\title{
Global existence and uniqueness for the inhomogeneous 1-Laplace evolution equation
}

Sergio Segura de León and Claudete M. Webler

\begin{abstract}
In this paper we introduce a new approach to the Dirichlet problem for the total variation flow in a bounded domain and analyze the associated inhomogeneous problem. We prove global existence and uniqueness for source data belonging to $L_{l o c}^{1}\left(0,+\infty ; L^{2}(\Omega)\right)$ and $L^{2}$-initial data. We compare solutions corresponding to different data as well as study the long-term behaviour of the solutions. We also show explicit examples of radial solutions.
\end{abstract}

Mathematics Subject Classification. 35K55, 35K20, 35K67, 35D30, 35K92.

Keywords. Inhomogeneous evolution equations, Singular diffusion equations, 1-Laplacian, Dirichlet problem, Total variation flow, $L^{2}$-initial data, $p$-Laplacian.

\section{Introduction}

Let $\Omega$ be a bounded open subset in $\mathbb{R}^{N}$ with a Lipschitz-continuous boundary $\partial \Omega$. Our aim is to study the following Dirichlet problem:

$$
\left\{\begin{aligned}
u^{\prime}-\operatorname{div}\left(\frac{D u}{|D u|}\right) & =f(x, t), & & \text { in } \Omega \times(0,+\infty) ; \\
u & =0, & & \text { on } \partial \Omega \times(0,+\infty) ; \\
u(x, 0) & =u_{0}(x), & & \text { in } \Omega ;
\end{aligned}\right.
$$

where data satisfy $f \in L_{l o c}^{1}\left(0,+\infty ; L^{2}(\Omega)\right)$ and $u_{0} \in L^{2}(\Omega)$ (here and in what follows $L_{l o c}^{1}(0,+\infty ; X)$ means $L^{1}(0, T ; X)$ for all $T>0$, where $X$ denotes a space of functions depending on the spatial variable $x)$. We will prove that there exists a unique solution $u \in L_{l o c}^{1}(0,+\infty ; B V(\Omega)) \cap C\left([0,+\infty) ; L^{2}(\Omega)\right)$ and will compare solutions corresponding to different data. The main results of this paper are collected in Corollaries 5.3-5.5. Moreover, we will show the 
long-term behaviour of a solution depending on the source datum $f$ and some explicit simple solutions will be given.

The homogeneous case of this problem (that is, when $f \equiv 0$ ) was first analyzed in [2] (see also [5]). Among the motivations to study this problem lies a variational approach for filling in regions of missing data in digital images (see [8]). It is introduced, in [2], a suitable concept of solution to this kind of problems, defining the expression $\frac{D u}{|D u|}$ and the boundary condition in a weak sense through a bounded vector field whose distributional divergence is regular enough. The solution is obtained by means of nonlinear semigroup methods: first an accretive operator is defined which leads to a mild solution of the evolution problem (via Crandall-Liggett's Theorem) and then this mild solution is characterized by means of a variational formulation.

The methods used in that pioneer paper were applied in a series of articles $[3,4,6,9]$ dealing with linear growth functionals (their results, and some other related, can be found in the book [5]). Nevertheless, these papers always analyze the homogeneous equation. To our knowledge, the only paper that handles the inhomogeneous case is [21]. Although there is some overlap with the results in Sect. 3 below, this overlap is not very much because the purpose of [21] is to study stability of $p$-Laplace evolution equations as $p$ goes to 1 . The techniques employed in [21] also rely on the theory of nonlinear semigroups. We point out that data in [21] belong to $L_{l o c}^{2}\left(0,+\infty ; L^{2}(\Omega)\right)$, so that our data are slightly more general and thus we highlight the Lebesgue space that is truly relevant in this context (see Corollaries 5.3, 5.5 and Theorem 6.1 below).

Of course, we may apply nonlinear semigroup methods to obtain a mild solution of the inhomogeneous equation, but the second part of the procedure (the characterization of the mild solution) would remain to be seen. Instead of the semigroup approach, we obtain our existence and uniqueness results in a straightforward way. Thus, this paper not only deals with the nonhomogeneous problem, but it also provides a new approach to the homogeneous one.

Two important tools used in our approach are a suitable Gronwall's inequality and Proposition 2.10 (see also Remark 3.2). Gronwall's lemma leads to a priori estimates involving the $L_{l o c}^{1}\left(0,+\infty ; L^{2}(\Omega)\right)$-norm which, as was already mentioned, we believe is the appropriate one to study the inhomogeneous problem in a variational setting. On the other hand, the expression (6) appearing in Proposition 2.10 allows us to compare different solutions and so provides a new procedure for uniqueness (without using the method of doubling variables). Moreover, both tools will be essential to prove Corollary 5.5 and to study the long-term behaviour of the solution, extending the results obtained for the homogeneous case in [3]. It should also be remarked that, in dealing with the time derivative in the approximating problems, it is necessary to apply the regularization with respect to time introduced in [14].

This paper is organized as follows. Section 2 is on Preliminaries. In Sect. 3 we introduce our concept of solution, which is slightly different of that given in [2]. Section 4 is devoted to prove existence for our problem with data in $L_{l o c}^{2}\left(0,+\infty ; L^{2}(\Omega)\right)$, while in Sect. 5 we deal with existence and uniqueness 
when data in $L_{l o c}^{1}\left(0,+\infty ; L^{2}(\Omega)\right)$ are taken. In Sect. 5 we also compare solutions corresponding to different data. Section 6 is concerned with large term behaviour of the solutions. In the last section we show some explicit solutions.

\section{Notation and preliminaries}

Throughout this paper let $\Omega$ represent a bounded open set in $\mathbb{R}^{N}, N \geq 1$. We assume that $\Omega$ has a Lipschitz-continuous boundary and denote by $\nu(x)$ the outer unit normal vector at $x \in \partial \Omega$, which exists $\mathcal{H}^{N-1}$-a.e.; here and in what follows $\mathcal{H}^{N-1}$ stands for the $(N-1)$-dimensional Hausdorff measure. For $q \in[1, \infty]$, the symbol $L^{q}(\Omega)$ denote the usual Lebesgue space and we will denote by $W_{0}^{1, q}(\Omega)$ the usual Sobolev space of measurable functions having weak derivative in $L^{q}(\Omega)$ and zero trace on $\partial \Omega$, see for instance [11]. Moreover, we will denote by $q^{\prime}$ Hölder's conjugate exponent of $q>1$, i.e., $\frac{1}{q}+\frac{1}{q^{\prime}}=1$. Finally, if $1 \leq q<N$, we will denote by $q^{*}=N q /(N-q)$ its Sobolev conjugate exponent.

Throughout this paper, $u^{\prime}$ denotes the derivative with respect to $t$, while div stands for the divergence with respect to $x$; the gradient with respect to $x$ will be denoted by $\nabla$ for functions in a Sobolev space and by $D$ when $B V$-functions are considered.

The class of functions $u \in L^{1}(\Omega)$ whose partial derivatives in the sense of distributions are measures with finite total variation in $\Omega$ is denoted by $B V(\Omega)$ and is called the space of functions of bounded variation in $\Omega$. The space $B V(\Omega)$ is endowed with the norm

$$
\|u\|_{B V(\Omega)}=\int_{\Omega}|D u|+\int_{\Omega}|u| d x
$$

where $|D u|$ is the total variation of the vector-valued measure $D u$. With this norm, $B V(\Omega)$ is a Banach space, which happens to be the dual of a Banach space denoted by $\Gamma(\Omega)$ (we refer to [1] where a characterization of the space $\Gamma(\Omega)$ can be found). An equivalent norm is given by

$$
u \mapsto \int_{\Omega}|D u|+\int_{\partial \Omega}|u| d \mathcal{H}^{N-1} .
$$

It defines a functional in $B V(\Omega)$ that is lower semi-continuous with respect to the $L^{1}(\Omega)$-convergence. Another functional we will use, that is lower semicontinuous in $L^{1}(\Omega)$, is given by

$$
u \mapsto \int_{\Omega} \varphi|D u|,
$$

where $\varphi \in C_{0}^{\infty}(\Omega)$ satisfies $\varphi \geq 0$. More information on functions of bounded variation can be found in [1] or [12].

If $T>0$, we write $Q_{T}=\Omega \times(0, T)$. For $r, q \in[1,+\infty]$, the spaces $L^{r}\left(0, T ; L^{q}(\Omega)\right), L^{r}\left(0, T ; W_{0}^{1, q}(\Omega)\right)$ and $L^{r}(0, T ; B V(\Omega))$ have obvious meanings. For the sake of brevity, if $X$ is a Banach space of functions depending on the spatial variable $x$, we shall write $u(x, t) \in L_{\mathrm{loc}}^{r}(0, \infty ; X)$ instead of 
" $u(x, t) \in L^{r}(0, \tau ; X)$ for every $\tau>0$ ". In this framework, fixed $t>0$, we often write " $u(t) \in X$ " to mean that the function $x \mapsto u(x, t)$ belongs to $X$.

We will need some concepts and results from [7] (see also [5, Appendix C]) to make sense of the dot product of a bounded vector field (whose divergence satisfies certain conditions) and the gradient of a $B V$-function, and so we may apply a Green's formula. This theory is studied in [10] as well, although from a different point of view. These tools were extended in [2] to treat evolution equations of the kind studied in the present paper. Nevertheless, our approach is slightly different, since our aim is to prove Proposition 2.10 below.

Given $\mathbf{z} \in L^{\infty}\left(\Omega ; \mathbb{R}^{N}\right)$, we denote by $\operatorname{div} \mathbf{z}$ the divergence of $\mathbf{z}$ in the sense of distributions, i.e.

$$
\langle\operatorname{div} \mathbf{z}, \varphi\rangle=-\int_{\Omega} \mathbf{z} \cdot \nabla \varphi d x
$$

for all $\varphi \in C_{0}^{\infty}(\Omega)$. Assume also that $\operatorname{div} \mathbf{z} \in L^{2}(\Omega)$. Then, following [7], we may define a weak trace on $\partial \Omega$ of the normal component of $\mathbf{z}$, that will be represented by $[\mathbf{z}, \nu]$. It holds that $[\mathbf{z}, \nu] \in L^{\infty}(\partial \Omega)$ and $\|[\mathbf{z}, \nu]\|_{\infty} \leq\|\mathbf{z}\|_{\infty}$. Moreover, we may characterize this function by the following property: for each $w \in W^{1,1}(\Omega) \cap L^{2}(\Omega)$

$$
\int_{\partial \Omega} w[\mathbf{z}, \nu] d \mathcal{H}^{N-1}=\int_{\Omega} w \operatorname{div} \mathbf{z} d x+\int_{\Omega} \mathbf{z} \cdot \nabla w d x
$$

holds true. On the other hand, given $v \in B V(\Omega) \cap L^{2}(\Omega)$ and $\mathbf{z} \in L^{\infty}\left(\Omega ; \mathbb{R}^{N}\right)$ such that $\operatorname{div} \mathbf{z} \in L^{2}(\Omega)$, we define the distribution

$$
(\mathbf{z}, D v): C_{0}^{\infty}(\Omega) \rightarrow \mathbb{R}
$$

by

$$
\langle(\mathbf{z}, D v), \varphi\rangle=-\int_{\Omega} v \mathbf{z} \cdot \nabla \varphi d x-\int_{\Omega} v \varphi \operatorname{div} \mathbf{z} d x,
$$

for all $\varphi \in C_{0}^{\infty}(\Omega)$. Observe that, under our hypotheses, every term of (2) has sense. In [7] the following result is proved.

Proposition 2.1. Let $\mathbf{z} \in L^{\infty}\left(\Omega ; \mathbb{R}^{N}\right)$ satisfy div $\mathbf{z} \in L^{2}(\Omega)$.

The distribution $(\mathbf{z}, D v)$ is actually a Radon measure with finite total variation.

The measures $(\mathbf{z}, D v)$ and its total variation $|(\mathbf{z}, D v)|$ are absolutely continuous with respect to $|D v|$ and

$$
\left|\int_{B}(\mathbf{z}, D v)\right| \leq \int_{B}|(\mathbf{z}, D v)| \leq\|\mathbf{z}\|_{L^{\infty}(U)} \int_{B}|D v|
$$

holds for every Borel set $B$ and every open set $U$ such that $B \subset U \subset \Omega$. Furthermore, the following generalized Green's formula holds:

$$
\int_{\Omega} v \operatorname{div}(\mathbf{z}) d x+\int_{\Omega}(\mathbf{z}, D v)=\int_{\partial \Omega}[\mathbf{z}, \nu] v d \mathcal{H}^{N-1},
$$

where $[\mathbf{z}, \nu]$ denotes the weak trace on $\partial \Omega$ of the normal component of $\mathbf{z}$. 
Given a Banach space $X$, we will write by $X^{*}$ its dual space. Thus, $B V(\Omega)^{*}$ stands for the dual space of $B V(\Omega)$ and $B V(\Omega)=\Gamma(\Omega)^{*}$.

When $\operatorname{div} \mathbf{z} \in B V(\Omega)^{*}$, we define the distribution

$$
(\mathbf{z}, D v): C_{0}^{\infty}(\Omega) \rightarrow \mathbb{R}
$$

by

$$
\langle(\mathbf{z}, D v), \varphi\rangle=-\int_{\Omega} v \mathbf{z} \cdot \nabla \varphi d x-\langle\operatorname{div} \mathbf{z}, v \varphi\rangle_{B V(\Omega)^{*}, B V(\Omega)},
$$

for all $\varphi \in C_{0}^{\infty}(\Omega)$. Since $v \in B V(\Omega) \subset L^{1}(\Omega), \varphi \in C_{0}^{\infty}(\Omega), \mathbf{z} \in L^{\infty}\left(\Omega ; \mathbb{R}^{N}\right)$ and $\operatorname{div} \mathbf{z} \in B V(\Omega)^{*}$, it follows that all terms of (4) are well-defined. Moreover, we may also define $[\mathbf{z}, \nu] \in L^{\infty}(\partial \Omega)$ satisfying (2) for all $w \in W^{1,1}(\Omega)$ (see [2] and [5]). In general, we do not know that a result similar to Proposition 2.1 holds. However, in [17, Theorems 5.1 and 5.2] it is proved that this type of results holds when div $\mathbf{z}$ belongs to the predual $\Gamma(\Omega)$ of $B V(\Omega)$.

If $\xi \in B V(\Omega)^{*}+L^{2}(\Omega)$, then we may consider $\xi \in W_{0}^{1,1}(\Omega)^{*}+L^{2}(\Omega)$ and so there exist $\mathbf{z} \in L^{\infty}\left(\Omega ; \mathbb{R}^{N}\right)$ and $f \in L^{2}(\Omega)$ satisfying

$$
\langle\xi, w\rangle=\int_{\Omega} \mathbf{z} \cdot \nabla w+\int_{\Omega} f w
$$

for all $w \in W_{0}^{1,1}(\Omega) \cap L^{2}(\Omega)$. In this sense, we may write

$$
\xi=\operatorname{div} \mathbf{z}+f \quad \text { in } \mathcal{D}^{\prime}(\Omega) .
$$

Obviously this decomposition need not be unique. Given a specific decomposition $\xi=\operatorname{div} \mathbf{z}+f$, it is straightforward that one can define the weak trace $[\mathbf{z}, \nu] \in L^{\infty}(\partial \Omega)$ and $\|[\mathbf{z}, \nu]\|_{\infty} \leq\|\mathbf{z}\|_{\infty}$ holds true. Nevertheless, we cannot prove that this vector field $\mathbf{z}$ appearing in the decomposition satisfies that the distribution given by (4) is a Radon measure. So, the following definition is necessary.

Definition 2.2. We say that

$$
\xi=\operatorname{div} \mathbf{z}+f
$$

is a regular decomposition of $\xi \in B V(\Omega)^{*}+L^{2}(\Omega)$ if

1. $\xi=\operatorname{div} \mathbf{z}+f$ in $\mathcal{D}^{\prime}(\Omega)$;

2. $(\mathbf{z}, D v)$ is a Radon measure for all $v \in B V(\Omega) \cap L^{2}(\Omega)$;

3. $\langle\xi, v\rangle+\int_{\Omega}(\mathbf{z}, D v)=\int_{\partial \Omega} v[\mathbf{z}, \nu] d \mathcal{H}^{N-1}+\int_{\Omega} f v d x$, for all $v \in B V(\Omega) \cap$ $L^{2}(\Omega)$.

Remark 2.3. If $\xi \in L^{2}(\Omega)$, then every decomposition $\xi=\operatorname{div} \mathbf{z}+f$, with $\mathbf{z} \in L^{\infty}\left(\Omega ; \mathbb{R}^{N}\right)$ and $f \in L^{2}(\Omega)$, is regular owing to Proposition 2.1.

Lemma 2.4. Assume that $\left(z_{\alpha}\right)_{\alpha \in I}$ is a net satisfying

1. $\mathbf{z}_{\alpha} \rightarrow \mathbf{z}{ }^{*}$-weakly in $L^{\infty}\left(\Omega ; \mathbb{R}^{N}\right)$;

2. div $\mathbf{z}_{\alpha} \rightarrow$ div $\mathbf{z}^{*}$-weakly in $B V(\Omega)^{*}+L^{2}(\Omega)$;

3. $\left(\mathbf{z}_{\alpha}, D v\right)$ is a Radon measure for all $v \in B V(\Omega) \cap L^{2}(\Omega)$;

4. for each $\alpha \in I$ a Green's formula holds 


$$
\left\langle\operatorname{div} \mathbf{z}_{\alpha}, v\right\rangle_{B V(\Omega) *, B V(\Omega)}+\int_{\Omega}\left(\mathbf{z}_{\alpha}, D v\right)=\int_{\partial \Omega} v\left[\mathbf{z}_{\alpha}, \nu\right] d \mathcal{H}^{N-1},
$$

for all $v \in B V(\Omega) \cap L^{2}(\Omega)$.

Then for all $v \in B V(\Omega) \cap L^{2}(\Omega)$ we obtain

1. $(\mathbf{z}, D v)$ is a Radon measure;

2. $\left(\mathbf{z}_{\alpha}, D v\right) \rightarrow(\mathbf{z}, D v){ }^{*}$-weakly as measures;

3. $\left[\mathbf{z}_{\alpha}, \nu\right] \rightarrow[\mathbf{z}, \nu]{ }^{*}$-weakly in $L^{\infty}(\partial \Omega)$;

4. it yields

$$
\langle\operatorname{div} \mathbf{z}, v\rangle_{B V(\Omega)^{*}, B V(\Omega)}+\int_{\Omega}(\mathbf{z}, D v)=\int_{\partial \Omega} v[\mathbf{z}, \nu] d \mathcal{H}^{N-1} .
$$

Proof. (1) and (2).

Fix $\varphi \in C_{0}^{\infty}(\Omega)$ and $v \in B V(\Omega) \cap L^{2}(\Omega)$. We have

$$
\left\langle\left(\mathbf{z}_{\alpha}, D v\right), \varphi\right\rangle=-\left\langle\operatorname{div} \mathbf{z}_{\alpha}, v \varphi\right\rangle-\int_{\Omega} v \mathbf{z}_{\alpha} \cdot \nabla \varphi d x .
$$

Taking the limit in $\alpha$, the net converges to

$$
-\langle\operatorname{div} \mathbf{z}, v \varphi\rangle_{B V(\Omega)^{*}, B V(\Omega)}-\int_{\Omega} v \mathbf{z} \cdot \nabla \varphi=\langle(\mathbf{z}, D v), \varphi\rangle
$$

wherewith (2) holds once we see that $(\mathbf{z}, D v)$ is a measure. Moreover, it follows from

$$
\left|\left\langle\left(\mathbf{z}_{\alpha}, D v\right), \varphi\right\rangle\right| \leq\|\varphi\|_{\infty}\left\|\mathbf{z}_{\alpha}\right\|_{\infty} \int_{\Omega}|D v| \leq C|| \varphi \|_{\infty} \int_{\Omega}|D v|
$$

that $|\langle(\mathbf{z}, D v), \varphi\rangle| \leq C\|\varphi\|_{\infty} \int_{\Omega}|D v|$ and so (1) holds.

(3) For all $w \in W^{1,1}(\Omega) \cap L^{2}(\Omega)$, it yields

$$
\left\langle\operatorname{div} \mathbf{z}_{\alpha}, w\right\rangle_{B V(\Omega)^{*}, B V(\Omega)}+\int_{\Omega} \mathbf{z}_{\alpha} \cdot \nabla w d x=\int_{\partial \Omega} w\left[\mathbf{z}_{\alpha}, \nu\right] d \mathcal{H}^{N-1} .
$$

Then

$$
\begin{array}{r}
\lim _{\alpha} \int_{\partial \Omega} w\left[\mathbf{z}_{\alpha}, \nu\right] d \mathcal{H}^{N-1}=\lim _{\alpha}\left\langle\operatorname{div} \mathbf{z}_{\alpha}, w\right\rangle_{B V(\Omega)^{*}, B V(\Omega)}+\int_{\Omega} \mathbf{z}_{\alpha} \cdot \nabla w d x \\
=\langle\operatorname{div} \mathbf{z}, w\rangle_{B V(\Omega)^{*}, B V(\Omega)}+\int_{\Omega} \mathbf{z} \cdot \nabla w d x=\int_{\partial \Omega} w[\mathbf{z}, \nu] d \mathcal{H}^{N-1}
\end{array}
$$

(4) Set $v \in B V(\Omega) \cap L^{2}(\Omega)$. It follows that

$$
\begin{aligned}
& \langle\operatorname{div} \mathbf{z}, v\rangle_{B V(\Omega) *, B V(\Omega)}+\int_{\Omega}(\mathbf{z}, D v) \\
& =\lim _{\alpha}\left\langle\operatorname{div} \mathbf{z}_{\alpha}, v\right\rangle_{B V(\Omega)^{*}, B V(\Omega)}+\int_{\Omega}\left(\mathbf{z}_{\alpha}, D v\right) \\
& =\lim _{\alpha} \int_{\partial \Omega} v\left[\mathbf{z}_{\alpha}, \nu\right] d \mathcal{H}^{N-1}=\int_{\partial \Omega} v[\mathbf{z}, \nu] d \mathcal{H}^{N-1} .
\end{aligned}
$$

Proposition 2.5. Every $\xi \in B V(\Omega)^{*}+L^{2}(\Omega)$ has a regular decomposition. 
Proof. By the Goldstine Theorem, the closed unit ball of the predual $\Gamma(\Omega)$ is dense in the closed unit ball of the space $B V(\Omega)^{*}$ with respect to the topology $\sigma\left(B V(\Omega)^{*}, B V(\Omega)\right)$. Thus we can write

$$
\xi=\lim _{\alpha} \operatorname{div} \mathbf{z}_{\alpha}+f
$$

with $\operatorname{div} \mathbf{z}_{\alpha} \in \Gamma(\Omega)$; moreover, the net $\left(\operatorname{div} \mathbf{z}_{\alpha}\right)_{\alpha \in I}$ is bounded in $\Gamma(\Omega)$. This condition of being bounded implies that $\left(\operatorname{div} \mathbf{z}_{\alpha}\right)_{\alpha}$ is bounded in $W^{-1, \infty}(\Omega)$ and $\left(\mathbf{z}_{\alpha}\right)_{\alpha}$ is bounded in $L^{\infty}\left(\Omega ; \mathbb{R}^{N}\right)$. Then

$$
\begin{aligned}
\operatorname{div} \mathbf{z}_{\alpha}+f \rightarrow \xi & { }^{*} \text {-weakly in } B V(\Omega)^{*} \\
\mathbf{z}_{\alpha} \rightarrow \mathbf{z} & { }^{*} \text {-weakly in } L^{\infty}(\Omega) .
\end{aligned}
$$

Given $\varphi \in W_{0}^{1,1}(\Omega) \cap L^{2}(\Omega)$, it yields

$$
\langle\xi, \varphi\rangle=\lim _{\alpha} \int_{\Omega} \mathbf{z}_{\alpha} \cdot \nabla \varphi d x+\int_{\Omega} f \varphi d x=\int_{\Omega} \mathbf{z} \cdot \nabla \varphi d x+\int_{\Omega} f \varphi d x
$$

and so

$$
\xi=\operatorname{div} \mathbf{z}+f \quad \text { en } \quad \mathcal{D}^{\prime}(\Omega) .
$$

Applying [17, Theorem 5.1], each $\left(\mathbf{z}_{\alpha}, D v\right)$ is a Radon measure satisfying a Green's formula. Then, by Lemma 2.4, the decomposition is regular.

To reach Proposition 2.10, we still have to define some concepts introduced in [2]. We denote by $L_{w}^{1}(0, T ; B V(\Omega))$ the space of all measurable maps

$$
v:[0, T] \rightarrow B V(\Omega)
$$

(that is, $t \in[0, T] \rightarrow\langle\phi, v(t)\rangle_{B V(\Omega)^{*}, B V(\Omega)}$ is measurable for all $\phi \in B V(\Omega)^{*}$ ) such that $\int_{0}^{T}\|v(t)\|_{B V(\Omega)} d t<\infty$.

Definition 2.6. Let $\Psi \in L^{1}(0, T ; B V(\Omega)) \cap L^{\infty}\left(0, T ; L^{2}(\Omega)\right)$.

We say that $\Psi$ admits a weak derivative in $L_{w}^{1}(0, T ; B V(\Omega)) \cap L^{\infty}\left(Q_{T}\right)$ if there exists $\Theta \in L_{w}^{1}(0, T ; B V(\Omega)) \cap L^{\infty}\left(Q_{T}\right)$ such that $\Psi(t)=\int_{0}^{t} \Theta(s) d s$, where the integral is taken as a Pettis integral.

Definition 2.7. Let $u \in C\left([0, T] ; L^{2}(\Omega)\right) \cap L^{1}(0, T ; B V(\Omega))$. We will say that $\xi \in L^{1}(0, T ; B V(\Omega))^{*}+L^{1}\left(0, T ; L^{2}(\Omega)\right)$ satisfying $\xi=\operatorname{div} \mathbf{z}+f$ in $\mathcal{D}^{\prime}\left(Q_{T}\right)$ is the time derivative of $u$ if

1. $\int_{0}^{T}\langle\xi(t), \Psi(t)\rangle d t=-\int_{0}^{T} \int_{\Omega} u(s, x) \Theta(s, x) d x d s$, for every function

$$
\Psi \in L^{1}(0, T ; B V(\Omega)) \cap L^{\infty}\left(0, T ; L^{2}(\Omega)\right)
$$

which admits a weak derivative $\Theta \in L_{w}^{1}(0, T ; B V(\Omega)) \cap L^{\infty}\left(0, T ; L^{2}(\Omega)\right)$ and having compact support in time;

2. For almost all $t \in(0, T), \xi(t)=\operatorname{div} \mathbf{z}(t)+f(t)$ is a regular decomposition.

Remark 2.8. We explicitly remark that the dual $L^{1}(0, T ; B V(\Omega))^{*}$ can be identify with $L_{w^{*}}^{\infty}\left(0, T ; B V(\Omega)^{*}\right)$, the space of all $\xi:[0, T] \rightarrow B V(\Omega)^{*}$ which are ${ }^{*}$-weakly measurable (that is, the function $t \mapsto\langle\xi(t), v\rangle$ is measurable for 
every $v \in B V(\Omega))$ and satisfy $\|\xi(t)\|_{B V(\Omega)^{*}} \in L^{\infty}(0, T)$ (see [19]). The duality is given by

$$
\langle\xi, v\rangle=\int_{0}^{T}\langle\xi(t), v(t)\rangle_{B V(\Omega)^{*}, B V(\Omega)} d t
$$

for all $\xi \in L_{w^{*}}^{\infty}\left(0, T ; B V(\Omega)^{*}\right)$ and all $v \in L^{1}(0, T ; B V(\Omega))$. Consequently the first term of the formula 1 . in the above definition makes sense.

Proposition 2.9. Let $\xi \in L^{1}(0, T ; B V(\Omega))^{*}+L^{1}\left(0, T ; L^{2}(\Omega)\right)$ be such that $\xi=$ div $\mathbf{z}+f$ is the time derivative of $u \in C\left([0, T] ; L^{2}(\Omega)\right) \cap L^{1}(0, T ; B V(\Omega))$. Then the real function $t \mapsto \int_{\Omega} u(t)^{2} d x$ is absolutely continuous in $(0, T)$ and

$$
\begin{aligned}
& \frac{1}{2}\left(\int_{\Omega} u(t)^{2} d x\right)^{\prime} \\
& \quad=-\int_{\Omega}(\mathbf{z}(t), D u(t))+\int_{\partial \Omega} u(t)[\mathbf{z}(t), \nu] d \mathcal{H}^{N-1}+\int_{\Omega} f(t) u(t) d x .
\end{aligned}
$$

Proof. Consider $\eta \in C_{0}^{\infty}(0, T)$ and let $\tau$ be small enough to perform the following manipulations:

$$
\begin{aligned}
- & \int_{0}^{T} \int_{\Omega} \frac{\eta(t-\tau)-\eta(t)}{-\tau} \frac{u(t)^{2}}{2} d x d t \\
& =\int_{0}^{T} \int_{\Omega} \frac{u(t)^{2} \eta(t-\tau)}{2 \tau} d x d t-\int_{0}^{T} \int_{\Omega} \frac{u(t)^{2} \eta(t)}{2 \tau} d x d t \\
& =\int_{0}^{T} \int_{\Omega} \frac{u(t+\tau)^{2} \eta(t)}{2 \tau} d x d t-\int_{0}^{T} \int_{\Omega} \frac{u(t)^{2} \eta(t)}{2 \tau} d x d t \\
& =\frac{1}{2} \int_{0}^{T} \int_{\Omega} \frac{u(t+\tau)^{2}-u(t)^{2}}{\tau} \eta(t) d x d t \\
& =\frac{1}{2} \int_{0}^{T} \int_{\Omega} \frac{(u(t+\tau)-u(t))(u(t+\tau)+u(t))}{\tau} \eta(t) d x d t
\end{aligned}
$$

Thus,

$$
\begin{aligned}
& -\int_{0}^{T} \int_{\Omega} \frac{\eta(t-\tau)-\eta(t) \frac{u(t)^{2}}{2} d x d t}{-\tau} \frac{1}{2}\left[\int_{0}^{T} \int_{\Omega} \frac{(u(t+\tau)-u(t))}{\tau} u(t+\tau) \eta(t) d x d t\right. \\
& \left.\quad+\int_{0}^{T} \int_{\Omega} \frac{(u(t+\tau)-u(t))}{\tau} u(t) \eta(t) d x d t\right] \\
& =\frac{1}{2}\left(I_{1}+I_{2}\right) .
\end{aligned}
$$

To compute $I_{2}$ we define

$$
\Psi^{\tau}(t)=\frac{1}{\tau} \int_{t-\tau}^{t} \eta(s) u(s) d s .
$$


Vol. 22 (2015) The inhomogeneous 1-Laplace evolution equation

Then, since $\xi$ is the time derivative of $u$, we have

$$
\begin{aligned}
\int_{0}^{T}\left\langle\Psi^{\tau}(t), \xi(t)\right\rangle d t & =-\int_{0}^{T} \int_{\Omega} \frac{\eta(t) u(t)-\eta(t-\tau) u(t-\tau)}{\tau} u(t) d x d t \\
& =-\int_{0}^{T} \int_{\Omega} \frac{\eta(t) u(t)^{2}}{\tau} d x d t+\int_{0}^{T} \int_{\Omega} \frac{u(t+\tau)}{\tau} u(t) \eta(t) d x d t \\
& =I_{2}
\end{aligned}
$$

so that

$$
\begin{aligned}
I_{2}= & \int_{0}^{T}\left\langle\Psi^{\tau}(t), \xi(t)\right\rangle d t \\
= & -\int_{0}^{T} \int_{\Omega}\left(\mathbf{z}(t), D \Psi^{\tau}(t)\right) d t \\
& +\int_{0}^{T} \int_{\partial \Omega} \Psi^{\tau}(t)[\mathbf{z}(t), \nu] d \mathcal{H}^{N-1} d t \\
& +\int_{0}^{T} \int_{\Omega} f \Psi^{\tau}(t) d x d t \\
= & -\int_{0}^{T} \int_{\Omega}\left(\frac{1}{\tau} \int_{t-\tau}^{t} \eta(s)(\mathbf{z}(t), D u(s)) d s\right) d t \\
& +\int_{0}^{T} \int_{\partial \Omega}\left(\frac{1}{\tau} \int_{t-\tau}^{t} \eta(s) u(s) d s\right)[\mathbf{z}(t), \nu] d \mathcal{H}^{N-1} d t \\
& +\int_{0}^{T} \int_{\Omega} f\left(\frac{1}{\tau} \int_{t-\tau}^{t} \eta(s) u(s) d s\right) d x d t .
\end{aligned}
$$

Observe that

$$
\begin{aligned}
\lim _{\tau \rightarrow 0} I_{2}= & -\int_{0}^{T} \int_{\Omega} \eta(t)(\mathbf{z}(t), D u(t)) d t \\
& +\int_{0}^{T} \int_{\Omega} \eta(t) u(t)[\mathbf{z}(t), \nu] d \mathcal{H}^{N-1} d t+\int_{0}^{T} \int_{\Omega} f \eta u d x d t
\end{aligned}
$$

On the other hand, to compute $I_{1}$, we define

$$
\chi^{\tau}(t)=\frac{1}{\tau} \int_{t-\tau}^{t} \eta(s) u(s+\tau) d s .
$$

Hence,

$$
\begin{gathered}
\int_{0}^{T}\left\langle\chi^{\tau}(t), \xi(t)\right\rangle d t=-\int_{0}^{T} \int_{\Omega} \frac{\eta(t) u(t+\tau)-\eta(t-\tau) u(t)}{\tau} u(t) d x d t \\
=-\int_{0}^{T} \int_{\Omega} \frac{\eta(t) u(t+\tau) u(t)}{\tau} d x d t+\int_{0}^{T} \int_{\Omega} \frac{\eta(t-\tau) u(t)^{2}}{\tau} d x d t \\
=-\int_{0}^{T} \int_{\Omega} \frac{\eta(t) u(t+\tau) u(t)}{\tau} d x d t+\int_{0}^{T} \int_{\Omega} \frac{\eta(t) u(t+\tau)^{2}}{\tau} d x d t
\end{gathered}
$$




$$
\begin{aligned}
& =\int_{0}^{T} \int_{\Omega} \frac{u(t+\tau)-u(t)}{\tau} u(t+\tau) \eta(t) d x d t \\
& =I_{1}
\end{aligned}
$$

Arguing as in the case of $I_{2}$, it yields

$$
\lim _{\tau \rightarrow 0} I_{1}=\lim _{\tau \rightarrow 0} I_{2} .
$$

Therefore, it follows from

$$
-\int_{0}^{T} \int_{\Omega} \frac{\eta(t-\tau)-\eta(t)}{-\tau} \frac{u(t)^{2}}{2} d x d t=\frac{1}{2}\left(I_{1}+I_{2}\right)
$$

that

$$
\begin{aligned}
- & \int_{0}^{T} \int_{\Omega} \eta^{\prime}(t) \frac{u(t)^{2}}{2} d x d t \\
= & -\int_{0}^{T} \int_{\Omega} \eta(t)(\mathbf{z}(t), D u(t)) d t+\int_{0}^{T} \int_{\partial \Omega} \eta(t) u(t)[\mathbf{z}(t), \nu] d \mathcal{H}^{N-1} d t \\
& +\int_{0}^{T} \int_{\Omega} f(t) \eta(t) u(t) d x d t
\end{aligned}
$$

One concludes that

$$
\left(\int_{\Omega} \frac{u(t)^{2}}{2} d x\right)^{\prime}=\int_{\Omega}(\mathbf{z}(t), D u(t))+\int_{\partial \Omega} u(t)[\mathbf{z}(t), \nu] d \mathcal{H}^{N-1}+\int_{\Omega} f(t) u(t) d x
$$

and, since the right hand side belongs to $L^{1}(0, T)$, the function $t \rightarrow \int_{\Omega} \frac{u(t)^{2}}{2} d x$ is absolutely continuous.

Proposition 2.10. Consider $u, v \in C\left([0, T] ; L^{2}(\Omega)\right) \cap L^{1}(0, T ; B V(\Omega))$ with time derivatives $\xi, \bar{\xi}$, respectively. So

$$
\xi, \bar{\xi} \in L^{1}(0, T ; B V(\Omega))^{*}+L^{1}\left(0, T ; L^{2}(\Omega)\right)
$$

and we have the decompositions

$$
\xi=\operatorname{div} \mathbf{z}+f \quad \text { and } \quad \bar{\xi}=\operatorname{div} \overline{\mathbf{z}}+\bar{f}
$$

where $\mathbf{z}, \overline{\mathbf{z}} \in L^{\infty}\left(\Omega ; \mathbb{R}^{N}\right)$ and $f, \bar{f} \in L^{1}\left(0, T ; L^{2}(\Omega)\right)$.

Then, for almost all $t \in[0, T]$, it holds

$$
\begin{aligned}
& \left(\int_{\Omega} u(t) v(t) d x\right)^{\prime}=-\int_{\Omega}(\mathbf{z}(t), D v(t))-\int_{\Omega}(\overline{\mathbf{z}}(t), D u(t)) \\
& \quad+\int_{\partial \Omega} u(t)[\overline{\mathbf{z}}(t), \nu] d \mathcal{H}^{N-1}+\int_{\partial \Omega} v(t)[\mathbf{z}(t), \nu] d \mathcal{H}^{N-1} \\
& \quad+\int_{\Omega} f(t) v(t) d x+\int_{\Omega} \bar{f}(t) u(t) d x
\end{aligned}
$$


Proof. Obviously $u+v$ has as time derivative $\xi+\bar{\xi}$. Furthermore, each of the functions $u, v$ and $u+v$ satisfies the respective equation (5), wherewith we deduce, for almost all $t \in(0, T)$,

$$
\begin{aligned}
& \left(\int_{\Omega} u(t) v(t) d x\right)^{\prime} \\
& =\left(\int_{\Omega} \frac{(u(t)+v(t))^{2}}{2} d x\right)^{\prime}-\left(\int_{\Omega} \frac{u(t)^{2}}{2} d x\right)^{\prime}-\left(\int_{\Omega} \frac{v(t)^{2}}{2} d x\right)^{\prime} \\
& =-\int_{\Omega}(\mathbf{z}+\overline{\mathbf{z}}, D(u+v))+\int_{\partial \Omega}(u+v)[\mathbf{z}+\overline{\mathbf{z}}, \nu] d \mathcal{H}^{N-1}+\int_{\Omega}(f+\bar{f})(u+v) d x \\
& \quad+\int_{\Omega}(\mathbf{z}, D u)-\int_{\partial \Omega} u[\mathbf{z}, \nu] d \mathcal{H}^{N-1}-\int_{\Omega} f u d x \\
& \quad+\int_{\Omega}(\overline{\mathbf{z}}, D v)-\int_{\partial \Omega} v[\overline{\mathbf{z}}, \nu] d \mathcal{H}^{N-1}-\int_{\Omega} \bar{f} v d x
\end{aligned}
$$

and simplifying it yields

$$
\begin{aligned}
& \left(\int_{\Omega} u(t) v(t) d x\right)^{\prime} \\
& =-\int_{\Omega}(\mathbf{z}, D v)-\int_{\Omega}(\overline{\mathbf{z}}, D u)+\int_{\partial \Omega} u[\overline{\mathbf{z}}, \nu] d \mathcal{H}^{N-1}+\int_{\partial \Omega} v[\mathbf{z}, \nu] d \mathcal{H}^{N-1} \\
& \quad+\int_{\Omega} f v d x+\int_{\Omega} \bar{f} u d x .
\end{aligned}
$$

Remark 2.11. Since the right hand side of (6) belongs to $L^{1}(0, T)$, the function given by

$$
t \mapsto \int_{\Omega} u(t) v(t) d x
$$

is absolutely continuous in $(0, T)$.

\section{Definition of solution to problem (1)}

We are now ready to introduce the concept of solution to problem (1). For each $T>0$, we will consider the following parabolic problem

$$
\left\{\begin{aligned}
u^{\prime}-\operatorname{div}\left(\frac{D u}{|D u|}\right) & =f(x, t), & & \text { in } \Omega \times(0, T) ; \\
u & =0, & & \text { on } \partial \Omega \times(0, T) ; \\
u(x, 0) & =u_{0}(x), & & \text { in } \Omega ;
\end{aligned}\right.
$$

Definition 3.1. Let $f \in L^{1}\left(0, T ; L^{2}(\Omega)\right)$ and $u_{0} \in L^{2}(\Omega)$.

We say that a function $u \in L^{1}(0, T ; B V(\Omega)) \cap C\left([0, T] ; L^{2}(\Omega)\right)$ is a solution to $(7)$ if there exist $\mathbf{z} \in L^{\infty}\left(Q_{T}\right)$ and $u^{\prime} \in L^{1}(0, T ; B V(\Omega))^{*}+$ $L^{1}\left(0, T ; L^{2}(\Omega)\right)$ such that for almost all $t \in[0, T]$ it holds 


$$
\begin{gathered}
u^{\prime}(t)=\operatorname{div} \mathbf{z}(t)+f(t) \quad \text { is a regular decomposition } \\
(\mathbf{z}(t), D u(t))=|D u(t)| \quad \text { as measures }
\end{gathered}
$$

and

$$
[\mathbf{z}(t), \nu] \in \operatorname{sign}(-u(t)) \quad \mathcal{H}^{N-1} \quad \text { a.e. on } \partial \Omega .
$$

Moreover, $u^{\prime}$ is the time derivative of $u$.

Given $f \in L_{l o c}^{1}\left(0,+\infty ; L^{2}(\Omega)\right)$ and $u_{0} \in L^{2}(\Omega)$, we say that a function $u \in L_{\text {loc }}^{1}(0,+\infty ; B V(\Omega)) \cap C\left([0,+\infty) ; L^{2}(\Omega)\right)$ is a solution to (1) if it is a solution to (7) for every $T>0$.

Remark 3.2. Proposition 2.10 implies that if $u$ is a solution to (7) in the sense of Definition 3.1, then it satisfies the following property: for almost all $t \in(0, T)$

$$
\begin{aligned}
& \left(\int_{\Omega} u(t) v(t) d x\right)^{\prime}=-\int_{\Omega}(\mathbf{z}(t), D v(t))-\int_{\Omega}(\overline{\mathbf{z}}(t), D u(t)) \\
& \quad+\int_{\partial \Omega} u(t)[\overline{\mathbf{z}}(t), \nu] d \mathcal{H}^{N-1}+\int_{\partial \Omega} v(t)[\mathbf{z}(t), \nu] d \mathcal{H}^{N-1} \\
& \quad+\int_{\Omega} f(t) v(t) d x+\int_{\Omega} \bar{f}(t) u(t) d x
\end{aligned}
$$

holds, whenever $v$ has $v^{\prime} \in L^{1}(0, T ; B V(\Omega))^{*}+L^{1}\left(0, T ; L^{2}(\Omega)\right)$ as time derivative and decomposition

$$
v^{\prime}=\operatorname{div} \overline{\mathbf{z}}+\bar{f} .
$$

In particular, taking $u=v$ and applying (9) and (10) it follows that

$$
\left(\int_{\Omega} u(t)^{2} d x\right)^{\prime}=-2 \int_{\Omega}|D u(t)|-2 \int_{\partial \Omega}|u(t)| d \mathcal{H}^{N-1}+2 \int_{\Omega} f(t) u(t) d x
$$

for almost all $t \in(0, T)$.

Remark 3.3. When the distributional derivative satisfies $u^{\prime}(t) \in L^{2}(\Omega)$ for almost all $t \in[0, T]$, the decomposition is always regular (see Remark 2.3). Moreover, if $u^{\prime} \in L^{2}\left(Q_{T}\right)$, then $u^{\prime}$ is the time derivative of $u$ since, given a function $\Psi \in L^{1}(0, T ; B V(\Omega)) \cap L^{\infty}\left(0, T ; L^{2}(\Omega)\right)$ which admits weak derivative $\Theta \in L_{w}^{1}(0, T ; B V(\Omega)) \cap L^{\infty}\left(0, T ; L^{2}(\Omega)\right)$ and has compact support in time, it holds (for $h>0$ )

$$
\begin{aligned}
\int_{0}^{T} & \left\langle u^{\prime}(t), \Psi(t)\right\rangle d t=\int_{0}^{T} \int_{\Omega} u^{\prime}(x, t) \Psi(x, t) d x d t \\
& =\lim _{h \rightarrow 0^{+}} \int_{0}^{T} \int_{\Omega} \frac{u(x, t+h)-u(x, t)}{h} \Psi(x, t) d x d t \\
& =-\lim _{h \rightarrow 0^{+}} \int_{0}^{T} \int_{\Omega} u(x, t) \frac{\Psi(x, t)-\Psi(x, t-h)}{h} d x d t
\end{aligned}
$$




$$
\begin{aligned}
& =-\lim _{h \rightarrow 0^{+}} \int_{0}^{T} \int_{\Omega} u(x, t)\left(\frac{1}{h} \int_{t-h}^{t} \Theta(x, \tau) d \tau\right) d x d t \\
& =-\int_{0}^{T} \int_{\Omega} u(x, t) \Theta(x, t) d x d t .
\end{aligned}
$$

An analogous argument works for $h<0$ and thus $u^{\prime}$ is the time derivative of $u$ in the sense of Definition 2.7.

Observe that it is enough that $u^{\prime} \in L^{2}(\Omega \times \operatorname{supp} \Psi)$; that is, it is sufficient to assume $u^{\prime} \in L^{2}\left(\delta, T-\delta ; L^{2}(\Omega)\right)$ for all $\delta>0$.

\section{Data in $L^{2}(\Omega \times(0, T))$}

Assume in this section that $f \in L^{2}(\Omega \times(0, T))$ and $u_{0} \in L^{2}(\Omega)$.

Theorem 4.1. For each $f \in L^{2}(\Omega \times(0, T))$ and each $u_{0} \in L^{2}(\Omega)$ there exists a solution to $(7)$ that satisfies $u^{\prime}(t) \in L^{2}(\Omega)$ for almost all $t \in[0, T]$ and

$$
\max _{t \in[0, T]}\|u(t)\|_{L^{2}(\Omega)} \leq\left\|u_{0}\right\|_{L^{2}(\Omega)}+\int_{0}^{T}\|f(t)\|_{L^{2}(\Omega)} d t .
$$

Proof. Consider the following approximating problems

$$
\left\{\begin{aligned}
u_{p}^{\prime}-\Delta_{p} u_{p} & =f(x, t), & & \text { in } \Omega \times(0, T) ; \\
u_{p} & =0, & & \text { on } \partial \Omega \times(0, T) ; \\
u_{p}(x, 0) & =u_{0}(x) & & \text { in } \Omega ;
\end{aligned}\right.
$$

where $1<p<2$.

It is well-known that there exists a solution $u_{p} \in L^{p}\left(0, T ; W_{0}^{1, p}(\Omega)\right) \cap$ $C\left([0, T] ; L^{2}(\Omega)\right)$ to this problem (if $p \geq 2$, the classical reference is [16], but in our case, it is a bit trickier: it can be applied the Galerkin approximation appearing in [15]). Hence, it holds

$$
\begin{aligned}
& \int_{\Omega} u_{p}(t) \varphi(t) d x-\int_{\Omega} u_{0} \varphi(0) d x-\int_{0}^{t} \int_{\Omega} u_{p} \varphi^{\prime} d x d \tau \\
& \quad+\int_{0}^{t} \int_{\Omega}\left|\nabla u_{p}\right|^{p-2} \nabla u_{p} \cdot \nabla \varphi d x d \tau=\int_{0}^{t} \int_{\Omega} f \varphi d x d \tau,
\end{aligned}
$$

for all $0<t<T$ and all $\varphi \in C^{1}\left([0, T] ; L^{2}(\Omega)\right) \cap L^{p}\left(0, T ; W_{0}^{1, p}(\Omega)\right)$. Moreover, $u_{p}$ can be used as test function. In doing so, applying also Hölder's inequality, we obtain

$$
\begin{aligned}
& \frac{1}{2} \int_{\Omega} u_{p}^{2}(x, t) d x-\frac{1}{2} \int_{\Omega} u_{0}^{2}(x) d x+\int_{0}^{t} \int_{\Omega}\left|\nabla u_{p}\right|^{p} d x d \tau=\int_{0}^{t} \int_{\Omega} f u_{p} d x d \tau \\
& \quad \leq \int_{0}^{t}\left(\int_{\Omega} f^{2}(x, \tau) d x\right)^{1 / 2}\left(\int_{\Omega} u_{p}^{2}(x, \tau) d x\right)^{1 / 2} d \tau .
\end{aligned}
$$

We proceed to divide the proof into several stages. 


\section{Step 1: A priori estimates}

Denoting $\zeta(t)=\left(\int_{\Omega} u_{p}^{2}(x, t) d x\right)^{1 / 2}$ and dropping a nonnegative term, (15) becomes

$$
\zeta(t)^{2} \leq \zeta(0)^{2}+2 \int_{0}^{t}\left(\int_{\Omega} f^{2}(x, \tau) d x\right)^{1 / 2} \zeta(\tau) d \tau .
$$

Applying a nonlinear version of Gronwall's Lemma (due to [18], see also [22, Theorem 1.2]), we deduce that

$$
\zeta(t) \leq \zeta(0)+\int_{0}^{t}\left(\int_{\Omega} f^{2}(x, \tau) d x\right)^{1 / 2} d \tau .
$$

Hence, the right hand side of (15) is bounded and it yields

$$
\frac{1}{2} \int_{\Omega} u_{p}^{2}(x, t) d x+\int_{0}^{t} \int_{\Omega}\left|\nabla u_{p}\right|^{p} d x d \tau \leq C
$$

where the constant $C$ only depends on $\left\|u_{0}\right\|_{L^{2}(\Omega)}$ and on $\|f\|_{L^{1}\left(0, T ; L^{2}(\Omega)\right)}$. Taking the supreme among every $0<t<T$, we conclude that

$$
\sup _{t \in[0, T]} \int_{\Omega} u_{p}^{2}(x, t) d x+\int_{Q_{T}}\left|\nabla u_{p}\right|^{p} d x d t \leq C .
$$

Thus, $\left(u_{p}\right)_{p}$ is bounded in $L^{\infty}\left(0, T ; L^{2}(\Omega)\right) \cap L^{1}\left(0, T ; W_{0}^{1,1}(\Omega)\right)$. Since $u_{p}^{\prime}=$ $\Delta_{p} u_{p}+f$, we have that $\left(u_{p}^{\prime}\right)_{p}$ is bounded in

$$
L^{p^{\prime}}\left(0, T ; W^{-1, p^{\prime}}(\Omega)\right)+L^{1}\left(0, T ; L^{2}(\Omega)\right) \subset L^{1}\left(0, T ; W^{-1,1}(\Omega)\right),
$$

Corollary 4 in [20] now implies that $u_{p}$ is compact in $L^{1}\left(Q_{T}\right)$. Therefore, there exists $u \in L^{1}\left(Q_{T}\right)$ and a subsequence (still denoted by $u_{p}$ ) satisfying

$$
\begin{gathered}
u_{p} \rightarrow u \quad \text { strongly in } L^{1}\left(Q_{T}\right) \\
u_{p}(x, t) \rightarrow u(x, t) \quad \text { a.e. in } Q_{T} .
\end{gathered}
$$

We deduce that

$$
u_{p}(t) \rightarrow u(t) \quad \text { in } L^{1}(\Omega) \text { for almost all } t \in(0, T) .
$$

Step 2: $u \in L^{1}(0, T ; B V(\Omega))$

By Young's inequality

$$
\int_{Q_{T}}\left|\nabla u_{p}(t)\right| d x d t \leq \frac{1}{p^{\prime}} T|\Omega|+\frac{1}{p} \int_{Q_{T}}\left|\nabla u_{p}(t)\right|^{p} d x d t \leq C,
$$

where $C$ only depends on the parameters of our problem. Fatou's Lemma implies

$$
\int_{0}^{T} \liminf _{p \rightarrow 1} \int_{\Omega}\left|\nabla u_{p}(t)\right| d x d t \leq C .
$$

Recalling (19), for almost all $t \in(0, T)$, it yields $u(t) \in B V(\Omega)$ and

$$
\int_{\Omega}|D u(t)| d x \leq \liminf _{p \rightarrow 1} \int_{\Omega}\left|\nabla u_{p}(t)\right| d x .
$$


From here and (20) one concludes that $u \in L^{1}(0, T ; B V(\Omega))$ and

$$
\int_{0}^{T} \int_{\Omega}|D u(t)| d x d t \leq \int_{0}^{T} \liminf _{p \rightarrow 1} \int_{\Omega}\left|\nabla u_{p}\right| d x d t .
$$

\section{Step 3: Existence of the vector field}

By (16) we know that

$$
\int_{Q_{T}}\left|\nabla u_{p}\right|^{p} d x d t \leq C
$$

and so, for all $1<s<p^{\prime}$, it holds

$$
\begin{aligned}
& \int_{Q_{T}}\left|\nabla u_{p}\right|^{s(p-1)} d x d t \leq\left(\int_{Q_{T}}\left|\nabla u_{p}\right|^{p} d x d t\right)^{\frac{s(p-1)}{p}}\left|Q_{T}\right|^{1-\frac{s(p-1)}{p}} \\
& \quad \leq C^{\frac{s(p-1)}{p}}\left|Q_{T}\right|^{1-\frac{s(p-1)}{p}} .
\end{aligned}
$$

Then, up to subsequences, we have that $\left(\left|\nabla u_{p}\right|^{p-2} \nabla u_{p}\right)_{p}$ weakly converges to $\mathbf{z}_{s}$ in $L^{s}\left(Q_{T} ; \mathbb{R}^{N}\right)$ for all $1 \leq s<+\infty$. Using a diagonal argument, we deduce that there exists a vector field $\mathbf{z} \in L^{s}\left(Q_{T} ; \mathbb{R}^{N}\right)$, where $1 \leq s<\infty$, such that

$$
\left|\nabla u_{p}\right|^{p-2} \nabla u_{p} \rightarrow \mathbf{z} \quad \text { weakly in each } L^{s}\left(Q_{T} ; \mathbb{R}^{N}\right), \quad 1 \leq s<+\infty .
$$

By letting $p \rightarrow 1$ in (21) we obtain

$$
\int_{Q_{T}}|\mathbf{z}|^{s} d x d t \leq\left|Q_{T}\right|
$$

that is, $\|\mathbf{z}\|_{s} \leq\left|Q_{T}\right|^{1 / s}$ for all $1 \leq s<\infty$. If now we let $s \rightarrow \infty$, then $\mathbf{z} \in L^{\infty}\left(Q_{T} ; \mathbb{R}^{N}\right)$ and $\|\mathbf{z}\|_{\infty} \leq 1$.

We next check the equation satisfied by $\mathbf{z}$. We take $\varphi \in C_{0}^{\infty}\left(Q_{T}\right)$ as test function in (14) obtaining

$$
-\int_{Q_{T}} u_{p} \varphi^{\prime} d x d t+\int_{Q_{T}}\left|\nabla u_{p}\right|^{p-2} \nabla u_{p} \cdot \nabla \varphi d x d t=\int_{Q_{T}} f \varphi d x d t .
$$

As $p$ goes to 1 , it leads to

$$
-\int_{Q_{T}} u \varphi^{\prime} d x d t+\int_{Q_{T}} \mathbf{z} \cdot \nabla \varphi d x d t=\int_{Q_{T}} f \varphi d x d t
$$

wherewith it satisfies

$$
u^{\prime}-\operatorname{div} \mathbf{z}=f(x, t), \quad \text { in } \mathcal{D}^{\prime}\left(Q_{T}\right) .
$$

Step 4: $u^{\prime}(t) \in L^{2}(\Omega)$ a.e.

For almost all $t>0$, we know that $u_{p}(t) \in W_{0}^{1, p}(\Omega)$ and $u(t) \in B V(\Omega)$. We fix one of these time values and denote it by $\delta>0$. We are applying the regularization with respect to time introduced in [14] for each fix $p>1$. For every $\nu>0$, we consider the solution to problem

$$
\left\{\begin{array}{l}
\frac{1}{\nu} u_{\nu}^{\prime}+u_{\nu}=u_{p} \\
u_{\nu}(\delta)=u_{p}(\delta)
\end{array}\right.
$$


Then $u_{\nu}^{\prime}, u_{\nu} \in L^{p}\left(0, T ; W_{0}^{1, p}(\Omega)\right)$ and $u_{\nu} \rightarrow u_{p}$ strongly in $L^{p}\left(0, T ; W_{0}^{1, p}(\Omega)\right)$ as $\nu \rightarrow \infty$. Taking $u_{\nu}^{\prime}$ as test we obtain

$$
\int_{\delta}^{t} \int_{\Omega} u_{p}^{\prime} u_{\nu}^{\prime} d x d \tau+\int_{\delta}^{t} \int_{\Omega}\left|\nabla u_{p}\right|^{p-2} \nabla u_{p} \cdot \nabla u_{\nu}^{\prime} d x d \tau=\int_{\delta}^{t} \int_{\Omega} f u_{\nu}^{\prime} d x d \tau
$$

Our aim is to use

$$
\int_{\delta}^{t} \int_{\Omega}\left(u_{\nu}^{\prime}\right)^{2} d x d \tau \leq \int_{\delta}^{t} \int_{\Omega} u_{p}^{\prime} u_{\nu}^{\prime} d x d \tau
$$

and this claim follows from

$$
\begin{aligned}
\int_{\delta}^{t} \int_{\Omega} u_{\nu}^{\prime}\left[u_{p}^{\prime}-u_{\nu}^{\prime}\right] d x d \tau & =\nu \int_{\delta}^{t} \int_{\Omega}\left(u_{p}-u_{\nu}\right)\left(u_{p}-u_{\nu}\right)^{\prime} d x d \tau \\
& =\frac{\nu}{2} \int_{\delta}^{t} \int_{\Omega}\left(\left(u_{p}-u_{\nu}\right)^{2}\right)^{\prime} d x d \tau \\
& =\frac{\nu}{2} \int_{\Omega}\left(u_{p}(t)-u_{p}(\delta)\right)^{2} d x d \tau \geq 0
\end{aligned}
$$

Thus, it follows from (24) that

$$
\begin{aligned}
& \int_{\delta}^{t} \int_{\Omega}\left(u_{\nu}^{\prime}\right)^{2} d x d \tau+\int_{\delta}^{t} \int_{\Omega}\left|\nabla u_{p}\right|^{p-2} \nabla u_{p} \cdot \nabla u_{\nu}^{\prime} d x d \tau \\
& \quad \leq \frac{1}{2} \int_{\delta}^{t} \int_{\Omega} f^{2} d x d \tau+\frac{1}{2} \int_{\delta}^{t} \int_{\Omega}\left(u_{\nu}^{\prime}\right)^{2} d x d \tau
\end{aligned}
$$

and so

$$
\begin{aligned}
& \frac{1}{2} \int_{\delta}^{t} \int_{\Omega}\left(u_{\nu}^{\prime}\right)^{2} d x d \tau+\int_{\delta}^{t} \int_{\Omega}\left|\nabla u_{p}\right|^{p-2} \nabla u_{p} \cdot \nabla u_{\nu}^{\prime} d x d \tau \\
& \quad \leq \frac{1}{2} \int_{\delta}^{t} \int_{\Omega} f^{2} d x d \tau .
\end{aligned}
$$

On the other hand, we also have

$$
\begin{aligned}
0 & \leq \nu \int_{\delta}^{t} \int_{\Omega}\left(\left|\nabla u_{p}\right|^{p-2} \nabla u_{p}-\left|\nabla u_{\nu}\right|^{p-2} \cdot \nabla u_{\nu}\right) \cdot \nabla\left(u_{p}-u_{\nu}\right) d x d \tau \\
& =\int_{\delta}^{t} \int_{\Omega}\left(\left|\nabla u_{p}\right|^{p-2} \nabla u_{p}-\left|\nabla u_{\nu}\right|^{p-2} \nabla u_{\nu}\right) \cdot \nabla u_{\nu}^{\prime} d x d \tau *
\end{aligned}
$$

so that

$$
\int_{\delta}^{t} \int_{\Omega}\left|\nabla u_{\nu}\right|^{p-2} \nabla u_{\nu} \cdot \nabla u_{\nu}^{\prime} d x d \tau \leq \int_{\delta}^{t} \int_{\Omega}\left|\nabla u_{p}\right|^{p-2} \nabla u_{p} \cdot \nabla u_{\nu}^{\prime} d x d \tau .
$$

Hence,

$$
\begin{aligned}
& \frac{1}{2} \int_{\delta}^{t} \int_{\Omega}\left(u_{\nu}^{\prime}\right)^{2} d x d \tau+\int_{\delta}^{t} \int_{\Omega}\left|\nabla u_{\nu}\right|^{p-2} \nabla u_{\nu} \cdot \nabla\left(u_{\nu}\right)^{\prime} d x d \tau \\
& \quad \leq \frac{1}{2} \int_{\delta}^{t} \int_{\Omega} f^{2} d x d \tau .
\end{aligned}
$$


Having in mind

$$
\begin{aligned}
& \int_{\delta}^{t} \int_{\Omega}\left|\nabla u_{\nu}\right|^{p-2} \nabla u_{\nu} \cdot \nabla\left(u_{\nu}\right)^{\prime} d x d \tau=\frac{1}{p} \int_{\delta}^{t}\left(\int_{\Omega}\left|\nabla u_{\nu}(t)\right|^{p}\right)^{\prime} d x d \tau \\
& \quad=\frac{1}{p} \int_{\Omega}\left|\nabla u_{\nu}(t)\right|^{p} d x-\frac{1}{p} \int_{\Omega}\left|\nabla u_{\nu}(\delta)\right|^{p} d x
\end{aligned}
$$

we deduce

$$
\frac{1}{2} \int_{\delta}^{t} \int_{\Omega}\left(u_{\nu}^{\prime}\right)^{2} d x d \tau \leq \frac{1}{2} \int_{\delta}^{t} \int_{\Omega} f^{2} d x d \tau+\frac{1}{p} \int_{\Omega}\left|\nabla u_{p}(\delta)\right|^{p} d x=C
$$

where $C$ does not depends on $\nu$. Then $u_{\nu}^{\prime}$ is bounded in $L^{2}\left(\delta, T ; L^{2}(\Omega)\right)$ and $\left(u_{\nu}(t)\right)^{\prime} \rightarrow \xi$ weakly in $L^{2}\left(\delta, T ; L^{2}(\Omega)\right)$; it is easy to see that $u_{\nu} \rightarrow u_{p}$ strongly in $L^{p}\left(0, T ; W_{0}^{1, p}(\Omega)\right)$ implies $\xi=u_{p}^{\prime}$ in the sense of distributions.

Taking $\nu \rightarrow \infty$ in (25), it yields

$$
\frac{1}{2} \int_{\delta}^{T} \int_{\Omega}\left(u_{p}^{\prime}\right)^{2} d x d t \leq \frac{1}{2} \int_{\delta}^{t} \int_{\Omega} f^{2} d x d \tau+\frac{1}{p} \int_{\Omega}\left|\nabla u_{p}(\delta)\right|^{p} d x,
$$

wherewith $\left(u_{p}^{\prime}\right)_{p}$ is bounded in $L^{2}\left(\delta, T ; L^{2}(\Omega)\right)$ and

$$
u_{p}^{\prime} \rightarrow u^{\prime} .
$$

Since this argument holds for almost all $\delta>0$, we conclude that $u^{\prime} \in L^{2}$ $\left(\delta, T ; L^{2}(\Omega)\right)$ for all $\delta>0$, thus we have check that $u^{\prime}(t) \in L^{2}(\Omega)$ for almost all $t \in[0, T]$.

Step 5: $u \in C\left([0, T] ; L^{2}(\Omega)\right)$

Since $u^{\prime} \in L^{2}\left(\delta, T ; L^{2}(\Omega)\right)$ for all $\delta>0$, by [20, Corollary 4], it follows that $u_{p} \rightarrow u$ in $C\left([\delta, T] ; L^{2}(\Omega)\right)$ for all $\delta>0$, and so $u \in C\left((0, T] ; L^{2}(\Omega)\right)$. As a consequence, $u(t)$ is well-defined for all $t>0$. Moreover, $u_{p}(\mathrm{t}) \rightarrow \mathrm{u}(\mathrm{t})$ in $\mathrm{L}^{2}(\Omega)$ for all $\mathrm{t}>0$. Let us see that $u$ is continuous at 0 as well.

On the one hand, for each $0<t<T$ it follows from (15) and (16) that

$$
\frac{1}{2} \int_{\Omega} u^{2}(t) d x-\frac{1}{2} \int_{\Omega} u_{0}^{2} d x \leq C \int_{0}^{t}\left(\int_{\Omega} f^{2} d x\right)^{1 / 2} d \tau .
$$

Since the function given by $t \mapsto\left(\int_{\Omega} f^{2}(t) d x\right)^{1 / 2}$ belongs to $L^{1}(0, T)$, we deduce that

$$
0=\lim _{t \rightarrow 0} \int_{0}^{t}\left(\int_{\Omega} f^{2} d x\right)^{1 / 2} d \tau
$$

Hence,

$$
\lim _{t \rightarrow 0} \int_{\Omega} u^{2}(t) d x \leq \int_{\Omega} u_{0}^{2} d x .
$$

On the other hand, consider $\varphi \in C_{0}^{\infty}(\Omega)$ and let $0<t<T$. Then (14) implies 


$$
\begin{aligned}
& \int_{\Omega} u(t) \varphi d x-\int_{\Omega} u_{0} \varphi d x-\int_{0}^{t} \int_{\Omega} u \varphi^{\prime} d x d \tau+\int_{0}^{t} \int_{\Omega}|\nabla u|^{p-2} \nabla u \cdot \nabla \varphi d x d \tau \\
& =\int_{0}^{t} \int_{\Omega} f \varphi d x d \tau
\end{aligned}
$$

Arguing as above, it yields

$$
\lim _{t \rightarrow 0} \int_{\Omega} u(t) \varphi d x=\int_{\Omega} u_{0} \varphi d x
$$

for all $\varphi \in C_{0}^{\infty}(\Omega)$. By density it may be extended to all $\varphi \in L^{2}(\Omega)$, wherewith $u(t) \rightarrow u_{0}$ weakly in $L^{2}(\Omega)$. As a consequence, $\int_{\Omega} u_{0}^{2} d x \leq \lim _{t \rightarrow 0}$ inf $\int_{\Omega} u^{2}(t) d x$ and then $\lim _{t \rightarrow 0} \int_{\Omega} u^{2}(t) d x=\int_{\Omega} u_{0}^{2} d x$

Since $\|u(t)\|_{2} \rightarrow\left\|u_{0}\right\|_{2}$ and $u(t) \rightarrow u_{0}$ weakly in $L^{2}(\Omega)$, Riesz-Radon's Theorem implies $u(t) \rightarrow u_{0}$ strongly in $L^{2}(\Omega)$. We get $u \in C\left([0, T] ; L^{2}(\Omega)\right)$.

Step 6: Equation $u^{\prime}(t)+\operatorname{div} \mathbf{z}(t)=f(t)$ in $\mathcal{D}^{\prime}(\Omega)$ holds for almost all $t$

Consider two functions $\phi \in C_{0}^{\infty}(\Omega)$ and $\psi \in C_{0}^{\infty}(0, T)$. Multiplying (23) by $\phi(x) \psi(t)$ and applying Green's formula, $\int_{\Omega} u_{0}^{2} d x \leq \lim _{t \rightarrow 0}$ inf $\int_{\Omega} u^{2}(t) d x$ it follows that

$$
\begin{aligned}
\int_{0}^{T} & \int_{\Omega} u^{\prime}(x, t) \phi(x) \psi(t) d x d t+\int_{0}^{T} \int_{\Omega} \psi(t) \mathbf{z}(x, t) \cdot \nabla \phi(x) d x d t \\
& =\int_{0}^{T} \int_{\Omega} f(x, t) \phi(x) \psi(t) d x d t .
\end{aligned}
$$

We already know that there exists $\delta>0$ such that supp $\psi \subset(\delta, T)$ and $u^{\prime} \in L^{2}\left(\delta, T ; L^{2}(\Omega)\right)$, so that, for almost all $t \in(0, T)$, it holds

$$
\int_{\Omega} u^{\prime}(x, t) \phi(x) d x+\int_{\Omega} \mathbf{z}(x, t) \cdot \nabla \phi(x) d x=\int_{\Omega} f(x, t) \phi(x) d x .
$$

We conclude that $u^{\prime}$ is the time derivative of $u$ (see Remark 3.3).

Step 7: For almost all $t>0$, we have $(\mathbf{z}(t), D u(t))=|D u(t)|$ as measures

Consider $\varphi(x, t)=\phi(x) \psi(t)$, with $\phi \in C_{0}^{\infty}(\Omega), \psi \in C_{0}^{\infty}(0, T)$ and $\phi, \psi \geq$ 0 . Denoting, as usual, the truncation at level $\pm k$ by $T_{k}(s)=\max \{-k, \min$ $\{k, s\}\}$, we take $T_{k}\left(u_{p}\right) \varphi$ as test function in (14):

$$
\begin{aligned}
\int_{Q_{T}} & T_{k}\left(u_{p}\right) u_{p}^{\prime} \phi \psi d x d t+\int_{Q_{T}}\left|\nabla T_{k}\left(u_{p}\right)\right|^{p} \phi \psi d x d t \\
& +\int_{Q_{T}} \psi T_{k}\left(u_{p}\right)\left|\nabla u_{p}\right|^{p-2} \nabla u_{p} \cdot \nabla \phi d x d t \\
= & \int_{Q_{T}} f T_{k}\left(u_{p}\right) \phi \psi d x d t .
\end{aligned}
$$

We want to let $p \rightarrow 1^{+}$. The right hand side offers no difficulty; we are analyzing the left one. By the lower semi-continuity of the total variation and Young's inequality, we obtain

$$
\int_{Q_{T}} \phi \psi\left|D T_{k}(u)\right| d t \leq \liminf _{p \rightarrow 1} \int_{Q_{T}} \phi \psi\left|\nabla T_{k}\left(u_{p}\right)\right| d x d t
$$




$$
\begin{aligned}
& \leq \liminf _{p \rightarrow 1}\left(\frac{1}{p} \int_{Q_{T}} \phi \psi\left|\nabla T_{k}\left(u_{p}\right)\right|^{p} d x d t+\frac{p-1}{p} \int_{Q_{T}} \phi \psi d x d t\right) \\
& =\liminf _{p \rightarrow 1} \frac{1}{p} \int_{Q_{T}} \phi \psi\left|\nabla T_{k}\left(u_{p}\right)\right|^{p} d x d t .
\end{aligned}
$$

On the other hand, choose $\delta>0$ such that sop $\psi \subset(\delta, T)$. Since $u_{p}^{\prime} \rightarrow u^{\prime}$ weakly in $L^{2}\left(\delta, T ; L^{2}(\Omega)\right)$ and $T_{k}\left(u_{p}\right) \rightarrow T_{k}(u)$ strongly in $L^{2}\left(\delta, T ; L^{2}(\Omega)\right)$, it yields

$$
\lim _{p \rightarrow 1} \int_{\delta}^{T} \int_{\Omega} T_{k}\left(u_{p}\right) u_{p}^{\prime} \phi \psi d x d t=\int_{\delta}^{T} \int_{\Omega} T_{k}(u) u^{\prime} \phi \psi d x d t .
$$

Finally, it follows from $\left|\nabla u_{p}\right|^{p-2} \nabla u_{p} \rightarrow \mathbf{z}$ weakly and $T_{k}\left(u_{p}\right) \rightarrow T_{k}(u)$ strongly in every $L^{s}\left(Q_{T} ; \mathbb{R}^{N}\right)$, where $1 \leq s<\infty$, that

$$
\lim _{p \rightarrow 1} \int_{Q_{T}} \psi T_{k}\left(u_{p}\right)\left|\nabla u_{p}\right|^{p-2} \nabla u_{p} \cdot \nabla \phi d x d t=\int_{Q_{T}} \psi T_{k}(u) \mathbf{z} \cdot \nabla \phi d x d t .
$$

Therefore, (26) becomes

$$
\begin{aligned}
& \int_{Q_{T}} T_{k}(u) u^{\prime} \phi \psi d x d t+\int_{Q_{T}} \phi \psi\left|D T_{k}(u)\right| d t+\int_{Q_{T}} \psi T_{k}(u) \mathbf{z} \cdot \nabla \phi d x d t \\
& \quad \leq \int_{Q_{T}} f T_{k}(u) \phi \psi d x d t .
\end{aligned}
$$

Now we may tend $k \rightarrow \infty$ and obtain

$$
\begin{aligned}
& \int_{Q_{T}} u u^{\prime} \phi \psi d x d t+\int_{Q_{T}} \phi \psi|D u| d t+\int_{Q_{T}} \psi u \mathbf{z} \cdot \nabla \phi d x d t \leq \int_{Q_{T}} f u \phi \psi d x d t \\
& =\int_{Q_{T}} u u^{\prime} \phi \psi d x d t-\int_{Q_{T}}(\operatorname{div} \mathbf{z}) u \phi \psi d x d t
\end{aligned}
$$

Simplifying it yields

$$
\int_{Q_{T}} \phi \psi|D u| d t+\int_{Q_{T}} \psi u \mathbf{z} \cdot \nabla \phi d x d t \leq-\int_{Q_{T}}(\operatorname{div} \mathbf{z}) u \phi \psi d x d t,
$$

and consequently

$$
\int_{0}^{T} \int_{\Omega} \phi \psi|D u| d t \leq \int_{0}^{T} \int_{\Omega} \phi \psi(\mathbf{z}, D u) d t
$$

for all $\phi \in C_{0}^{\infty}(\Omega)$ and all $\psi \in C_{0}^{\infty}(0, T)$, with $\phi, \psi \geq 0$. Thus, for almost all $t \in(0, T)$, it holds

$$
\int_{\Omega} \phi|D u(t)| \leq \int_{\Omega} \phi(\mathbf{z}, D u(t)) .
$$

Then $|D u(t)| \leq(\mathbf{z}(t), D u(t))$ as measures and, since $\|\mathbf{z}\|_{\infty} \leq 1$, we deduce (9). Step 8: For almost all $t>0$ it holds $[\mathbf{z}(t), \nu] \in \operatorname{sign}(-u(t)), \mathcal{H}^{N-1}$-a.e. on $\partial \Omega$

Fix $\delta>0$. Our starting point is derived from (13) by taking $u_{p}$ as test function:

$$
\int_{\delta}^{T} \int_{\Omega} u_{p} u_{p}^{\prime} d x d t+\int_{\delta}^{T} \int_{\Omega}\left|\nabla u_{p}\right|^{p} d x d t=\int_{\delta}^{T} \int_{\Omega} f u_{p} d x d t
$$


It follows from Young's inequality that

$$
\begin{aligned}
& \int_{\delta}^{T} \int_{\Omega}\left|\nabla u_{p}\right| d x d t \leq \frac{1}{p} \int_{\delta}^{T} \int_{\Omega}\left|\nabla u_{p}\right|^{p} d x d t+\frac{p-1}{p}|\Omega| T \\
& \quad=\frac{1}{p}\left(\int_{\delta}^{T} \int_{\Omega} f u_{p} d x d t-\int_{\delta}^{T} \int_{\Omega} u_{p}^{\prime} u_{p} d x d t\right)+\frac{p-1}{p}|\Omega| T .
\end{aligned}
$$

Letting $p \rightarrow 1$, we deduce

$$
\begin{aligned}
& \int_{\delta}^{T} \int_{\Omega}|D u|+\int_{\delta}^{T} \int_{\partial \Omega}|u| d \mathcal{H}^{N-1} d t \\
& \quad \leq \liminf _{p \rightarrow 1}\left[\frac{1}{p}\left(\int_{\delta}^{T} \int_{\Omega} f u_{p} d x d t-\int_{\delta}^{T} \int_{\Omega} u_{p}^{\prime} u_{p} d x d t\right)+\frac{p-1}{p}|\Omega| T\right] \\
& \quad=\int_{\delta}^{T} \int_{\Omega} f u d x d t-\int_{\delta}^{T} \int_{\Omega} u^{\prime} u d x d t=-\int_{\delta}^{T} \int_{\Omega} u \operatorname{div} \mathbf{z} d x d t .
\end{aligned}
$$

Applying Green's formula, we get

$$
\begin{aligned}
& \int_{\delta}^{T} \int_{\Omega}|D u| d t+\int_{\delta}^{T} \int_{\partial \Omega}|u| d \mathcal{H}^{N-1} d t \\
& \quad \leq \int_{\delta}^{T} \int_{\Omega}(\mathbf{z}, D u) d t-\int_{\delta}^{T} \int_{\partial \Omega} u[\mathbf{z}, \nu] d \mathcal{H}^{N-1} d t
\end{aligned}
$$

and this implies

$$
\int_{\delta}^{T} \int_{\partial \Omega}|u| d \mathcal{H}^{N-1} d t+\int_{\delta}^{T} \int_{\partial \Omega} u[\mathbf{z}, \nu] d \mathcal{H}^{N-1} d t \leq 0
$$

owing to $|D u|=(\mathbf{z}, D u)$. On account of $\|[\mathbf{z}, \nu]\|_{\infty} \leq\|\mathbf{z}\|_{\infty} \leq 1$, it follows that, for almost all $t \in(\delta, T),[\mathbf{z}(t), \nu] u(t)+|u(t)|=0, \mathcal{H}^{N-1}$-a.e. on $\partial \Omega$. In the end, since $\delta>0$ is arbitrary, we deduce (10).

Step 9: $u^{\prime}$ is the time derivative of $u$

It is enough to have in mind that $u \in L^{2}\left(\delta, T ; L^{2}(\Omega)\right)$ for all $\delta>0$ and Remark 3.3. We conclude that $u$ is a solution to $(7)$ in the sense of Definition 3.1 .

We will next deduce that the solution we have found satisfies a comparison principle.

Proposition 4.2. Let $u_{0}, v_{0} \in L^{2}(\Omega)$ and $f, g \in L^{2}\left(0, T ; L^{2}(\Omega)\right)$, and denote by $u$ (respectively, $v$ ) a solution to (7) with data $u_{0}$ and $f$ (respectively, $v_{0}$ and $g)$.

If $f \leq g$ and $u_{0} \leq v_{0}$, then $u(t) \leq v(t)$ for all $t>0$.

Proof. We represent by $\mathbf{z}_{1}$ the vector field associated to solution $u$ and by $\mathbf{z}_{2}$ the corresponding to solution $v$.

We know that Eq. (8), both for $u$ and for $v$, holds for almost all $t>0$. Choose one of these $t$, multiply both equations $(8)$ by $(u(t)-v(t))^{+}$and 
subtract them to get

$$
\begin{aligned}
& \int_{\Omega}(u(t)-v(t))^{+}(u(t)-v(t))^{\prime} d x+\int_{\Omega}\left(\mathbf{z}_{1}(t)-\mathbf{z}_{2}(t), D(u(t)-v(t))^{+}\right) \\
& =\int_{\partial \Omega}(u(t)-v(t))^{+}\left[\mathbf{z}_{1}(t)-\mathbf{z}_{2}(t), \nu\right] d \mathcal{H}^{N-1} \\
& \quad+\int_{\Omega}(f(t)-g(t))(u(t)-v(t))^{+} d x
\end{aligned}
$$

Observe first that all terms, except the first one on the left hand side, define real functions belonging to $L^{1}(0, T)$, so do the remaining term.

We are analyzing the sign of the terms in (29). Using (9) to both solutions, it yields $\left(\mathbf{z}_{1}(t)-\mathbf{z}_{2}(t), D(u(t)-v(t))\right) \geq 0$ as measure. Then the RadonNikodým derivative of this measure with respect $|D(u(t)-v(t))|$, say $\theta$, is a nonnegative function. So, applying [7, Proposition 2.8], we deduce that

$$
\left(\mathbf{z}_{1}(t)-\mathbf{z}_{2}(t), D(u(t)-v(t))^{+}\right)=\theta\left|D(u(t)-v(t))^{+}\right| \geq 0, \quad \text { as measure. }
$$

Hence, $\int_{\Omega}\left(\mathbf{z}_{1}(t)-\mathbf{z}_{2}(t), D(u(t)-v(t))^{+}\right) \geq 0$.

It follows from $(10)$ that $\int_{\partial \Omega}(u(t)-v(t))^{+}\left[\mathbf{z}_{1}(t)-\mathbf{z}_{2}(t), \nu\right] d \mathcal{H}^{N-1} \leq 0$. Finally, our hypotheses imply $\int_{\Omega}(f(t)-g(t))(u(t)-v(t))^{+} d x \leq 0$. Therefore, Eq. (29) becomes

$$
\left(\int_{\Omega}\left[(u(t)-v(t))^{+}\right]^{2} d x\right)^{\prime} \leq 0, \quad \text { for almost all } t>0 .
$$

On the other hand, we already have seen that the left hand term defines an integrable function, so that $t \mapsto \int_{\Omega}\left[(u(t)-v(t))^{+}\right]^{2} d x$ is an absolutely continuous function. Therefore, we obtain

$$
\int_{\Omega}\left[(u(t)-v(t))^{+}\right]^{2} d x \leq \int_{\Omega}\left[\left(u_{0}-v_{0}\right)^{+}\right]^{2} d x=0, \quad \text { for all } t>0,
$$

from where the result follows.

\section{Data in $L^{1}\left(0, T ; L^{2}(\Omega)\right)$}

In this section we will prove the main existence and uniqueness results for problem (1).

Theorem 5.1. For each $f \in L^{1}\left(0, T ; L^{2}(\Omega)\right)$ and each $u_{0} \in L^{2}(\Omega)$ there exists a solution to (7) according to Definition 3.1. Moreover, it satisfies

$$
\max _{t \in[0, T]}\|u(t)\|_{L^{2}(\Omega)} \leq\left\|u_{0}\right\|_{L^{2}(\Omega)}+\int_{0}^{T}\|f(t)\|_{L^{2}(\Omega)} d t .
$$

Proof. Consider a sequence $\left(f_{n}\right) \subset L^{2}\left(0, T ; L^{2}(\Omega)\right)$ satisfying

$$
f_{n} \rightarrow f \quad \text { strongly in } \quad L^{1}\left(0, T ; L^{2}(\Omega)\right) .
$$


By Theorem 4.1, for each $n$ there exists $u_{n}$ solution to problem

$$
\left\{\begin{aligned}
u_{n}^{\prime}-\operatorname{div}\left(\frac{D u_{n}}{\left|D u_{n}\right|}\right) & =f_{n}(x, t), & & \text { in } \Omega \times(0, T) ; \\
u_{n} & =0, & & \text { on } \partial \Omega \times(0, T) ; \\
u_{n}(x, 0) & =u_{0}(x) & & \text { in } \Omega .
\end{aligned}\right.
$$

Thereupon for each $n$ we have associated vector fields $\mathbf{z}_{n}$ and, for almost all $t>0$, it holds

$$
u_{n}^{\prime}(t)-\operatorname{div} \mathbf{z}_{n}(t)=f_{n}(t) \quad \text { en } \quad \mathcal{D}^{\prime}(\Omega) .
$$

Proceeding as in Theorem 4.1, we split the proof in several steps.

\section{Step 1: A priori estimates}

Taking $u_{n}$ as test function, for almost all $t \in(0, T)$ we get

$$
\begin{aligned}
& \frac{1}{2}\left(\int_{\Omega} u_{n}^{2}(t) d x\right)^{\prime}+\int_{\partial \Omega}\left|u_{n}(t)\right| d \mathcal{H}^{N-1}+\int_{\Omega}\left|D u_{n}(t)\right|=\int_{\Omega} f_{n}(t) u_{n}(t) d x \\
& \quad \leq\left(\int_{\Omega}\left|f_{n}(t)\right|^{2} d x\right)^{\frac{1}{2}}\left(\int_{\Omega}\left|u_{n}(t)\right|^{2} d x\right)^{\frac{1}{2}}
\end{aligned}
$$

Writing $\zeta_{n}(t)=\left(\int_{\Omega}\left|u_{n}(t)\right|^{2} d x\right)^{\frac{1}{2}}$ we obtain

$$
\zeta_{n}(t)^{2} \leq \zeta(0)^{2}+2 \int_{0}^{t}\left(\int_{\Omega}\left|f_{n}(x, \tau)\right|^{2} d x\right)^{\frac{1}{2}} \zeta_{n}(\tau) d \tau
$$

From Gronwall's Lemma (see [22, Theorem 1.2]) we deduce that

$$
\left(\int_{\Omega}\left|u_{n}(t)\right|^{2} d x\right)^{\frac{1}{2}} \leq\left(\int_{\Omega}\left|u_{0}\right|^{2} d x\right)^{\frac{1}{2}}+\int_{0}^{T}\left(\int_{\Omega}\left|f_{n}(x, \tau)\right|^{2} d x\right)^{\frac{1}{2}} d \tau \leq C,
$$

the boundedness due to the strong convergence of $f_{n} \rightarrow f$ in $L^{1}\left(0, T ; L^{2}(\Omega)\right)$.

So we deduce

$$
\max _{t \in[0, T]} \int_{\Omega} u_{n}^{2}(t) d x+2 \int_{0}^{T} \int_{\partial \Omega}\left|u_{n}(t)\right| d \mathcal{H}^{N-1} d t+2 \int_{0}^{T} \int_{\Omega}\left|D u_{n}(t)\right| d t \leq C .
$$

Hence, the sequence $\left(u_{n}\right)_{n}$ is bounded in $L^{\infty}\left(0, T ; L^{2}(\Omega)\right) \cap L^{1}(0, T ; B V(\Omega))$ and $\left(u_{n}^{\prime}\right)_{n}$ is bounded in the space $L^{\infty}\left(0, T ; W^{-1, \infty}(\Omega)\right)+L^{1}\left(0, T ; L^{2}(\Omega)\right) \subset$ $L^{1}\left(0, T ; W^{-1,2}(\Omega)\right)$. By [20, Corollary 4$]$, there exists a subsequence (not relabeled) such that $u_{n}$ converges in $L^{1}\left(Q_{T}\right)$; that is, there exists $u \in L^{1}\left(Q_{T}\right)$ such that

$$
\begin{aligned}
u_{n} & \rightarrow u \quad \text { in } L^{1}\left(Q_{T}\right) \\
u_{n}(x, t) & \rightarrow u(x, t) \quad \text { a.e. in } Q_{T} .
\end{aligned}
$$

Arguing as in Theorem 4.1, we deduce that $u \in L^{1}(0, T ; B V(\Omega))$ and

$$
\int_{0}^{T} \int_{\Omega}|D u(t)| d t \leq \int_{0}^{T} \liminf _{n \rightarrow \infty} \int_{\Omega}\left|D u_{n}(t)\right| d t
$$

\section{Step 2: Existence of the vector field}


It follows from $\left\|\mathbf{z}_{n}(t)\right\|_{\infty} \leq 1$ for all $n \in \mathbb{N}$ and almost all $t \in[0, T]$, that there exists $\mathbf{z} \in L^{\infty}\left(\Omega ; \mathbb{R}^{N}\right)$ such that

$$
\mathbf{z}_{n} \rightarrow^{*} \mathbf{z} \quad \text { weakly in } L^{\infty}\left(Q_{T}, \mathbb{R}^{N}\right) .
$$

If $\varphi \in C_{0}^{\infty}\left(Q_{T}\right)$, then

$$
-\int_{0}^{T} \int_{\Omega} u_{n} \varphi^{\prime} d x d t+\int_{0}^{T} \int_{\Omega} \mathbf{z}_{n} . \nabla \varphi d x d t=\int_{0}^{T} \int_{\Omega} f_{n} \varphi d x d t .
$$

Tending $n$ to $\infty$ we get

$$
-\int_{0}^{T} \int_{\Omega} u \varphi^{\prime} d x d t+\int_{0}^{T} \int_{\Omega} \mathbf{z} \cdot \nabla \varphi d x d t=\int_{0}^{T} \int_{\Omega} f \varphi d x d t
$$

and so

$$
u^{\prime}-\operatorname{div} \mathbf{z}=f \quad \text { in } \quad \mathcal{D}^{\prime}\left(Q_{T}\right) .
$$

Step 3: $u \in C\left([0, T] ; L^{2}(\Omega)\right)$

We next check that $u_{n} \rightarrow u$ in $C\left([0, T] ; L^{2}(\Omega)\right)$ and to this end, it is enough to see that $\left(u_{n}\right)_{n=1}^{\infty}$ is a Cauchy sequence.

Taking $u_{n}(t)-u_{m}(t)$ in $(31)$ for $n$ and for $m$ lead to

$$
\begin{aligned}
\int_{\Omega} & u_{n}^{\prime}(t)\left(u_{n}(t)-u_{m}(t)\right) d x+\int_{\Omega}\left(\mathbf{z}_{n}(t), D\left(u_{n}(t)-u_{m}(t)\right)\right) \\
& -\int_{\partial \Omega}\left(u_{n}(t)-u_{m}(t)\right)\left[\mathbf{z}_{n}(t), \nu\right] d \mathcal{H}^{N-1} \\
= & \int_{\Omega} f_{n}(t)\left(u_{n}(t)-u_{m}(t)\right) d x
\end{aligned}
$$

and

$$
\begin{aligned}
\int_{\Omega} & u_{m}^{\prime}(t)\left(u_{n}(t)-u_{m}(t)\right) d x+\int_{\Omega}\left(\mathbf{z}_{m}(t), D\left(u_{n}(t)-u_{m}(t)\right)\right) \\
& -\int_{\partial \Omega}\left(u_{n}(t)-u_{m}(t)\right)\left[\mathbf{z}_{m}(t), \nu\right] d \mathcal{H}^{N-1} \\
= & \int_{\Omega} f_{m}(t)\left(u_{n}(t)-u_{m}(t)\right) d x .
\end{aligned}
$$

Subtracting both expressions yields

$$
\begin{aligned}
& \frac{1}{2}\left(\int_{\Omega}\left(u_{n}(t)-u_{m}(t)\right)^{2} d x\right)^{\prime}+\int_{\Omega}\left(\left(\mathbf{z}_{n}(t)-\mathbf{z}_{m}(t)\right), D\left(u_{n}(t)-u_{m}(t)\right)\right. \\
& \quad-\int_{\partial \Omega}\left(u_{n}(t)-u_{m}(t)\right)\left[\mathbf{z}_{n}(t)-\mathbf{z}_{m}(t), \nu\right] d \mathcal{H}^{N-1} \\
& \quad=\int_{\Omega}\left(f_{n}(t)-f_{m}(t)\right)\left(u_{n}(t)-u_{m}(t)\right) d x .
\end{aligned}
$$

Dropping two nonnegative terms and integrating with respect to $t$, we obtain

$$
\frac{1}{2} \int_{\Omega}\left(u_{n}(t)-u_{m}(t)\right)^{2} d x \leq \int_{Q_{T}}\left|f_{n}(t)-f_{m}(t)\right|\left|u_{n}(t)-u_{m}(t)\right| d x d t
$$


Now the right hand side tends to 0 since $f_{n} \rightarrow f$ in $L^{1}\left(0, T ; L^{2}(\Omega)\right)$ and $u_{n}$ is bounded in $L^{\infty}\left(0, T ; L^{2}(\Omega)\right)$. We conclude that $\left(u_{n}\right)_{n=1}^{\infty}$ is a Cauchy sequence in $L^{\infty}\left(0, T ; L^{2}(\Omega)\right)$ and so $u \in C\left([0, T] ; L^{2}(\Omega)\right)$. Therefore, the function $u(t)$ is well-defined for all $t>0$.

Step 4: The equation holds in the sense of distributions

For every $w \in B V(\Omega) \cap L^{2}(\Omega)$, Green's formula implies

$$
\begin{aligned}
\int_{\Omega} u_{n}^{\prime}(t) w d x & =\int_{\Omega} \operatorname{div} \mathbf{z}_{n}(t) w d x+\int_{\Omega} f_{n}(t) w d x \\
& =-\int_{\Omega}\left(\mathbf{z}_{n}(t), D w\right)+\int_{\partial \Omega}\left[\mathbf{z}_{n}(t), \nu\right] w d \mathcal{H}^{N-1}+\int_{\Omega} f_{n}(t) w d x d t .
\end{aligned}
$$

Thus,

$$
\left|\int_{\Omega}\left(u_{n}^{\prime}(t)-f_{n}(t)\right) w d x\right| \leq \int_{\Omega}|D w|+\int_{\partial \Omega}|w| d \mathcal{H}^{N-1} \leq\|w\|_{B V(\Omega)} .
$$

Then $\left(u_{n}^{\prime}-f_{n}\right)_{n \in \mathbb{N}}$ is bounded in $\left(L^{1}(0, T ; B V(\Omega))^{*}\right.$. As a consequence, there exist $\xi \in\left(L^{1}(0, T ; B V(\Omega))^{*}+L^{1}\left(0, T ; L^{2}(\Omega)\right)\right.$ and a subnet $\left(u_{\alpha}^{\prime}\right)_{\alpha \in I}$ of the sequence $\left(u_{n}^{\prime}\right)_{n \in \mathbb{N}}$ such that $\left(u_{\alpha}^{\prime}-f_{\alpha}\right)_{\alpha \in I}$ converges to $\xi-f$ in the weak-* topology, being $I$ a directed set.

Given $\eta \in \mathcal{D}\left(Q_{T}\right)$, since $\eta \in L^{1}(0, T ; B V(\Omega)) \cap L^{\infty}\left(0, T ; L^{2}(\Omega)\right)$, it follows that

$$
\begin{aligned}
\langle\xi, \eta\rangle & =\lim _{\alpha \in I}\left\langle u_{\alpha}^{\prime}, \eta\right\rangle \\
& =\lim _{\alpha \in I} \int_{0}^{T}\left\langle u_{\alpha}^{\prime}(t), \eta(t)\right\rangle d t \\
& =\lim _{\alpha \in I} \int_{0}^{T} \int_{\Omega} u_{\alpha}^{\prime}(t) \eta(t) d x d t \\
& =\lim _{\alpha \in I} \int_{0}^{T} \int_{\Omega} \operatorname{div}\left(\mathbf{z}_{\alpha}(t)\right) \eta(t) d x d t+\lim _{\alpha} \int_{0}^{T} \int_{\Omega} f_{\alpha}(t) \eta(t) d x d t \\
& =-\lim _{\alpha \in I} \int_{0}^{T} \int_{\Omega} \mathbf{z}_{\alpha}(t) . \nabla \eta(t) d x d t+\lim _{\alpha} \int_{0}^{T} \int_{\Omega} f_{\alpha}(t) \eta(t) d x d t \\
& =-\int_{0}^{T} \int_{\Omega} \mathbf{z}(t) . \nabla \eta(t) d x d t+\int_{0}^{T} \int_{\Omega} f(t) \eta(t) d x d t \\
& =\langle\operatorname{div}(\mathbf{z})+f, \eta\rangle
\end{aligned}
$$

Hence,

$$
\xi=\operatorname{div}(\mathbf{z})+f \quad \text { en } \quad \mathcal{D}^{\prime}\left(Q_{T}\right) .
$$

On the other hand, if we take $\eta(t, x)=\phi(t) \psi(x)$, with $\phi \in C_{0}^{\infty}(0, T)$ and $\psi \in C_{0}^{\infty}(\Omega)$, the same computations used in the proof of Theorem 4.1 can be performed and so

$$
\xi(t)=\operatorname{div}(\mathbf{z}(t))+f(t) \quad \text { en } \quad \mathcal{D}^{\prime}(\Omega) \quad \text { a.e. } \quad t \in(0, T) .
$$

Step 5: $\xi$ is the time derivative of $u$ in the sense of Definition 2.7 
Let $\Psi \in L^{1}(0, T ; B V(\Omega)) \cap L^{\infty}\left(0, T ; L^{2}(\Omega)\right)$ admit a weak derivative $\Theta \in$ $L_{w}^{1}(0, T ; B V(\Omega)) \cap L^{\infty}\left(Q_{T}\right)$, i.e.,

$$
\Psi(t)=\int_{0}^{t} \Theta(s) d s
$$

the integral being taken as a Pettis integral.

We begin with the identify

$$
\int_{0}^{T}\langle\xi(t), \Psi(t)\rangle d t=\lim _{\alpha \in I} \int_{0}^{T}\left\langle u_{\alpha}^{\prime}(t), \Psi(t)\right\rangle d t,
$$

which holds because $\left(u_{\alpha}^{\prime}-f_{\alpha}\right)_{\alpha \in I}$ converges to $\xi-f$ in the weak-* topology and $f_{\alpha} \rightarrow f$ in $L^{1}\left(0, T ; L^{2}(\Omega)\right)$.

Now,

$$
\begin{aligned}
\int_{0}^{T}\left\langle u_{\alpha}^{\prime}(t), \Psi(t)\right\rangle d t & =\lim _{h} \int_{0}^{T} \int_{\Omega} \Psi(t) \frac{u_{\alpha}(t+h)-u_{\alpha}(t)}{h} d x d t \\
& =\lim _{h} \int_{0}^{T} \int_{\Omega} \frac{\Psi(t-h)-\Psi(t)}{h} u_{\alpha}(t) d x d t \\
& =-\lim _{h} \int_{0}^{T} \int_{\Omega}\left(\frac{1}{h} \int_{t-h}^{t} \Theta(s) d s\right) u_{\alpha}(t) d x d t \\
& =-\int_{0}^{T} \int_{\Omega} \Theta(t, x) u_{\alpha}(t, x) d x d t
\end{aligned}
$$

Taking the limit in $\alpha$ in the above expression, it yields

$$
\int_{0}^{t}\langle\xi(t), \Psi(t)\rangle d t=-\int_{0}^{T} \int_{\Omega} \Theta(t, x) u(t, x) d x d t .
$$

Having in mind $\xi(t)=\operatorname{div}(\mathbf{z}(t))+f(t)$ in $\mathcal{D}^{\prime}(\Omega)$ for almost all $t \in[0, T]$ and since the hypotheses of Lemma 2.4 holds for $\left(\mathbf{z}_{\alpha}(t)\right)_{\alpha \in I}$, we deduce that $\xi(t)=\operatorname{div} \mathbf{z}(t)+f(t)$ is a regular decomposition. Thus $\xi$ is the time derivative of $u$ in the sense of Definition 2.7.

Proposition 5.2. Given $f \in L^{1}\left(0, T ; L^{2}(\Omega)\right)$ and $u_{0} \in L^{2}(\Omega)$ there exists at most a solution to (7) in the sense of Definition 3.1 .

Proof. Assume that $u_{1}$ are $u_{2}$ are solutions to problem (7) in the sense of Definition 3.1, where $u_{1}^{\prime}=\operatorname{div} \mathbf{z}_{1}+f$ and $u_{2}^{\prime}=\operatorname{div} \mathbf{z}_{2}+f$. Then $\left(u_{1}-u_{2}\right)^{\prime}=$ $\operatorname{div}\left(\mathbf{z}_{1}-\mathbf{z}_{2}\right)$. Applying (6) to each of these solutions it holds, for almost all $t \in(0, T)$,

$$
\begin{aligned}
& \left(\int_{\Omega} u_{1}(t)\left(u_{1}(t)-u_{2}(t)\right) d x\right)^{\prime} \\
& =-\int_{\Omega}\left(\mathbf{z}_{1}(t), D\left(u_{1}(t)-u_{2}(t)\right)\right)-\int_{\Omega}\left(\mathbf{z}_{1}(t)-\mathbf{z}_{2}(t), D u_{1}(t)\right) \\
& \quad+\int_{\partial \Omega} u_{1}(t)\left[\mathbf{z}_{1}(t)-\mathbf{z}_{2}(t), \nu\right] d \mathcal{H}^{N-1}+\int_{\partial \Omega}\left(u_{1}(t)-u_{2}(t)\right)\left[\mathbf{z}_{1}(t), \nu\right] d \mathcal{H}^{N-1} \\
& \quad+\int_{\Omega} f(t)\left(u_{1}(t)-u_{2}(t)\right) d x
\end{aligned}
$$


and

$$
\begin{aligned}
& \left(\int_{\Omega} u_{2}(t)\left(u_{1}(t)-u_{2}(t)\right) d x\right)^{\prime} \\
& =-\int_{\Omega}\left(\mathbf{z}_{2}(t), D\left(u_{1}(t)-u_{2}(t)\right)\right)-\int_{\Omega}\left(\mathbf{z}_{1}(t)-\mathbf{z}_{2}(t), D u_{2}(t)\right) \\
& \quad+\int_{\partial \Omega} u_{2}(t)\left[\mathbf{z}_{1}(t)-\mathbf{z}_{2}(t), \nu\right] d \mathcal{H}^{N-1}+\int_{\partial \Omega}\left(u_{1}(t)-u_{2}(t)\right)\left[\mathbf{z}_{2}(t), \nu\right] d \mathcal{H}^{N-1} \\
& \quad+\int_{\Omega} f(t)\left(u_{1}(t)-u_{2}(t)\right) d x
\end{aligned}
$$

Subtracting both expression, we obtain

$$
\left(\int_{\Omega}\left(u_{1}(t)-u_{2}(t)\right)^{2} d x\right)^{\prime}=0 .
$$

Therefore,

$$
\int_{\Omega}\left(u_{1}(t)-u_{2}(t)\right)^{2} d x=\int_{\Omega}\left(u_{0}-u_{0}\right)^{2} d x=0, \quad \forall t \in[0, T] .
$$

and so $u_{1}(t)=u_{2}(t)$ for all $t \in[0, T]$.

As a consequence of Theorem 5.1 and Proposition 5.2, taking into account inequality (30), we obtain the existence of a unique global solution to (1) in the sense of Definition 3.1. Details on regularity are supplied in the next section.

Corollary 5.3. Given $f \in L_{\text {loc }}^{1}\left(0,+\infty ; L^{2}(\Omega)\right)$ and $u_{0} \in L^{2}(\Omega)$ there exists a unique $u \in L_{\text {loc }}^{1}(0,+\infty ; B V(\Omega)) \cap C\left([0,+\infty) ; L^{2}(\Omega)\right)$ which is a global solution to (1) in the sense of Definition 3.1. Moreover, this solution satisfies

$$
\max _{t \in[0, T]}\|u(t)\|_{L^{2}(\Omega)} \leq\left\|u_{0}\right\|_{L^{2}(\Omega)}+\int_{0}^{T}\|f(t)\|_{L^{2}(\Omega)} d t .
$$

for all $T>0$.

We conclude this section by relating solutions corresponding to different data.

Corollary 5.4. Let $u_{0}, v_{0} \in L^{2}(\Omega)$ and $f, g \in L_{\text {loc }}^{1}\left(0,+\infty ; L^{2}(\Omega)\right)$, and denote by $u$ (respectively, $v$ ) a solution to $(7)$ with data $u_{0}$ and $f$ (respectively, $v_{0}$ and $g)$.

If $f \leq g$ and $u_{0} \leq v_{0}$, then $u(t) \leq v(t)$ for all $t>0$.

Proof. It is enough to have in mind the uniqueness of solutions and apply Proposition 4.2 to the approximate solutions in Theorem 5.1 to obtain that the solutions we found in this result satisfy the required inequality for all $t>0$.

As a consequence of Corollary 5.4, we obtain that nonnegative data imply nonnegative solutions. 
Corollary 5.5. Let $u_{0}, v_{0} \in L^{2}(\Omega)$ and $f, g \in L_{l o c}^{1}\left(0,+\infty ; L^{2}(\Omega)\right)$, and denote by $u$ (respectively, $v$ ) a solution to $(7)$ with data $u_{0}$ and $f$ (respectively, $v_{0}$ and $g)$.

Then, for all $t>0$,

$$
\|u(t)-v(t)\|_{L^{2}(\Omega)} \leq\left\|u_{0}-v_{0}\right\|_{L^{2}(\Omega)}+\int_{0}^{t}\|f(\tau)-g(\tau)\|_{L^{2}(\Omega)} d \tau .
$$

Proof. Fix $t>0$. Arguing as in Proposition 5.2 and applying (6) to each of solutions $u$ and $v$ we deduce that the map $t \mapsto \int_{\Omega}(u(t)-v(t))^{2} d x$ is absolutely continuous and it holds, for almost all $\tau \in(0, t)$,

$$
\left(\int_{\Omega}(u(\tau)-v(\tau))^{2} d x\right)^{\prime} \leq 2 \int_{\Omega}(f(\tau)-g(\tau))(u(\tau)-v(\tau)) d x .
$$

So, using Hölder's inequality and integrating in $(0, t)$, we get

$$
\begin{aligned}
& \|u(t)-v(t)\|_{L^{2}(\Omega)}^{2} \\
& \quad \leq\left\|u_{0}-v_{0}\right\|_{L^{2}(\Omega)}^{2}+2 \int_{0}^{t}\|f(\tau)-g(\tau)\|_{L^{2}(\Omega)}\|u(\tau)-v(\tau)\|_{L^{2}(\Omega)} d \tau .
\end{aligned}
$$

Therefore, Gronwall's inequality [22, Theorem 1.2] gives us estimate (33).

\section{Long-term behaviour}

In this section, we will study the long-term behaviour of the solutions to (1). We recall that in the homogeneous case, it has been shown in [13] that the solutions stabilize as $t \rightarrow+\infty$ by converging to 0 . This result was improved in [3] by showing that there is a finite extinction time. We turn to study what happens in the inhomogeneous case.

Theorem 6.1. 1 . If $f \in L^{1}\left(0,+\infty ; L^{2}(\Omega)\right)$, then the global solution to problem (1) satisfies $u \in C\left(\left[0,+\infty\left[; L^{2}(\Omega)\right) \cap L^{1}(0,+\infty ; B V(\Omega))\right.\right.$. Moreover, there exists a sequence $t_{n} \rightarrow+\infty$ and there exists $v \in L^{2}(\Omega)$ such that $u\left(t_{n}\right) \rightarrow v$ weakly in $L^{2}(\Omega)$.

2. If $f \in L^{\infty}\left(0,+\infty ; L^{2}(\Omega)\right)$, then it holds $u \in W^{1, \infty}\left(0,+\infty ; L^{2}(\Omega)\right) \cap$ $L^{\infty}(0,+\infty ; B V(\Omega))$. Furthermore, there exists a sequence $t_{n} \rightarrow+\infty$ and there exists $v \in B V(\Omega)$ such that $u\left(t_{n}\right) \rightarrow v^{*}$-weakly in $B V(\Omega)$.

Proof. (1) By (32) we have that, for all $t \geq 0$, the global solution satisfies

$$
\begin{aligned}
& \frac{1}{2} \int_{\Omega} u(t)^{2} d x+\int_{0}^{t} \int_{\partial \Omega}|u(\tau)| d \mathcal{H}^{N-1} d \tau+\int_{0}^{t} \int_{\Omega}|D u(\tau)| d \tau \\
& \leq \frac{1}{2} \int_{\Omega} u_{0}^{2} d x+\left(\int_{\Omega} u_{0}^{2} d x\right)^{1 / 2} \int_{0}^{t}\left(\int_{\Omega} f(x, \tau) d x\right)^{1 / 2} d \tau \\
& \quad+\int_{0}^{t}\left(\int_{\Omega} f(x, \tau) d x\right)^{1 / 2}\left[\int_{0}^{\tau}\left(\int_{\Omega} f(x, \sigma) d x\right)^{1 / 2} d \sigma\right] d \tau
\end{aligned}
$$


Thus, if $f \in L^{1}\left(0,+\infty ; L^{2}(\Omega)\right)$, then

$$
\begin{aligned}
& \frac{1}{2} \int_{\Omega} u(t)^{2} d x+\int_{0}^{t} \int_{\partial \Omega}|u(\tau)| d \mathcal{H}^{N-1} d \tau+\int_{0}^{t} \int_{\Omega}|D u(\tau)| d \tau \\
& \quad \leq \frac{1}{2}\left\|u_{0}\right\|_{L^{2}(\Omega)}^{2}+\left\|u_{0}\right\|_{L^{2}(\Omega)}\|f\|_{L^{1}\left(0,+\infty ; L^{2}(\Omega)\right)}+\|f\|_{L^{1}\left(0,+\infty ; L^{2}(\Omega)\right)}^{2}
\end{aligned}
$$

for all $t \geq 0$. It follows that $u \in L^{\infty}\left(0,+\infty ; L^{2}(\Omega)\right) \cap L^{1}(0,+\infty ; B V(\Omega))$ and, as a consequence of the boundedness of $\int_{\Omega} u(t)^{2} d x$, there exists $v \in L^{2}(\Omega)$ such that, up to subsequences, $u(t) \rightarrow v$ weakly in $L^{2}(\Omega)$.

(2) It is a consequence of the following estimate that holds for almost all $t>0$ :

$$
\begin{aligned}
& \frac{1}{2}\left(\int_{\Omega} u(t)^{2} d x\right)^{\prime}+\int_{\partial \Omega}|u(t)| d \mathcal{H}^{N-1}+\int_{\Omega}|D u(t)| \leq \int_{\Omega}|f(t) u(t)| d x \\
& \quad \leq \frac{1}{2} \int_{\Omega} f(t)^{2} d x+\frac{1}{2} \int_{\Omega} u(t)^{2} d x \leq C .
\end{aligned}
$$

The norm of the datum $f$ in $L_{l o c}^{1}\left(0,+\infty ; L^{2}(\Omega)\right)$ is not the only relevant norm for the problem (7). Indeed, as in the associated elliptic problem, the dual norm plays an important role as well.

Theorem 6.2. Assume that $f \in L_{l o c}^{1}\left(0,+\infty ; L^{2}(\Omega)\right) \cap L^{\infty}\left(0,+\infty ; W^{-1, \infty}(\Omega)\right)$.

1. If $\|f\|_{L^{\infty}\left(0,+\infty ; W^{-1, \infty}(\Omega)\right)} \leq 1$, then the norm $\|u(t)\|_{L^{2}(\Omega)}$ defines a nonincreasing function. Moreover, there exist a sequence $t_{n} \rightarrow+\infty$ and $v \in L^{2}(\Omega)$ such that $u\left(t_{n}\right) \rightarrow v$ weakly in $L^{2}(\Omega)$.

2. If $\|f\|_{L^{\infty}\left(0,+\infty ; W^{-1, \infty}(\Omega)\right)}<1$, then $u(t) \rightarrow 0{ }^{*}$-weakly in $B V(\Omega)$ and weakly in $L^{2}(\Omega)$.

Proof. (1) To begin, we note that, for almost all $t>0, f(t) \in L^{2}(\Omega) \cap$ $\left.W^{-1, \infty}(\Omega)\right)$, so there exists $\mathbf{w} \in L^{\infty}(\Omega \times(0,+\infty))$ such that $f(t)=\operatorname{div} \mathbf{w}(t)$ a.e. Hence, for these $t$ it holds $\|\mathbf{w}(t)\|_{L^{\infty}(\Omega)}=\|f\|_{W^{-1, \infty}(\Omega)} \leq 1$ and $(\mathbf{w}(t)$, $D u(t))$ is a Radon measure. From the equation we then deduce

$$
\begin{aligned}
& \frac{1}{2}\left(\int_{\Omega} u(t)^{2} d x\right)^{\prime}+\int_{\partial \Omega}|u(t)| d \mathcal{H}^{N-1}+\int_{\Omega}|D u(t)|=\int_{\Omega} u(t) \operatorname{div} \mathbf{w}(t) d x \\
& \quad=\int_{\partial \Omega} u(t)[\mathbf{w}(t), \nu] d \mathcal{H}^{N-1}-\int_{\Omega}(\mathbf{w}(t), D u(t)) \\
& \quad \leq \int_{\partial \Omega}|u(t)| d \mathcal{H}^{N-1}+\int_{\Omega}|D u(t)| .
\end{aligned}
$$

Therefore, $\left(\int_{\Omega} u(t)^{2} d x\right)^{\prime} \leq 0$ for almost all $t>0$ and the norm $\|u(t)\|_{L^{2}(\Omega)}$ is nonincreasing. As a consequence, $\|u(t)\|_{L^{2}(\Omega)} \leq\left\|u_{0}\right\|_{L^{2}(\Omega)}$ for all $t>0$ and, up to subsequences, $(u(t))_{t>0}$ weakly converges in $L^{2}(\Omega)$.

We point out that, since the norm is nonnegative and nonincreasing, it follows that $\lim _{t \rightarrow+\infty}\|u(t)\|_{L^{2}(\Omega)}^{\prime}=0$. 
(2) Assume now that $\|f\|_{L^{\infty}\left(0,+\infty ; W^{-1, \infty}(\Omega)\right)}=1-\epsilon$, for some $\epsilon>0$. Arguing as above, we obtain that

$$
\frac{1}{2}\left(\int_{\Omega} u(t)^{2} d x\right)^{\prime}+\epsilon \int_{\partial \Omega}|u(t)| d \mathcal{H}^{N-1}+\epsilon \int_{\Omega}|D u(t)| \leq 0
$$

i.e., if we disregard a nonnegative term,

$$
\|u(t)\|_{B V(\Omega)} \leq \frac{1}{2 \epsilon}\left\|u_{0}\right\|_{L^{2}(\Omega)}^{2} .
$$

It yields that there exists $v \in B V(\Omega)$ such that, up to subsequences, $u(t) \rightarrow v$ *-weakly in $B V(\Omega)$. On the other hand, it follows from

$$
\int_{\partial \Omega}|u(t)| d \mathcal{H}^{N-1}+\int_{\Omega}|D u(t)| \leq-\frac{1}{2 \epsilon}\left(\|u(t)\|_{L^{2}(\Omega)}^{2}\right)^{\prime} \rightarrow 0,
$$

and applying the lower semi-continuity, that

$$
\int_{\partial \Omega}|v| d \mathcal{H}^{N-1}+\int_{\Omega}|D v|=0 .
$$

Thus $v \equiv 0$.

In the case $\|f\|_{L^{\infty}\left(0,+\infty ; W^{-1, \infty}(\Omega)\right)}<1$, a further remark is in order. If $\lim _{t \rightarrow+\infty}\|u(t)\|_{L^{2}(\Omega)}=0$, then by Riesz-Radon's Theorem we obtain that $u(t) \rightarrow 0$ strongly in $L^{2}(\Omega)$ as $t \rightarrow+\infty$.

\section{Explicit solutions}

In this section we present examples of radial solutions to problem (1) in a simple framework. Let $\Omega=B_{R}(0)$ be the open ball centered at 0 and having radius $R$. We choose data having the form $f(x, t)=g(t) \chi_{B_{r}(0)}(|x|)$ and $u_{0}(x)=$ $\alpha_{0} \chi_{B_{r}(0)}(|x|)$, with $0<r<R$. We, consequently, look for solutions $u(x, t)=$ $\alpha(t) \chi_{B_{r}(0)}(|x|)$, with $\alpha(0)=\alpha_{0}$. In what follows we assume that $\alpha_{0}>0$ and $g \in$ $L_{l o c}^{1}(0,+\infty)$ is nonnegative. According to our results and taking into account $|D u(x, t)|=\alpha(t)\left|D \chi_{B_{r}(0)}(|x|)\right|$, we deduce that $\alpha \in L_{l o c}^{1}(0,+\infty) \cap C([0,+\infty))$ and $\alpha^{2}$ is absolutely continuous on each bounded interval.

To search the solution, we apply the energy identity (11). Simplifying it yields

$$
\frac{1}{2}\left(\alpha^{2}(t)\right)^{\prime}\left|B_{r}(0)\right|=-\alpha(t) \mathcal{H}^{N-1}\left(\partial B_{r}(0)\right)+\alpha(t) g(t)\left|B_{r}(0)\right|, \quad t>0 .
$$

We deduce that, for a given $t>0$, either $\alpha(t)=0$ or $\alpha^{\prime}(t)=-\frac{N}{r}+g(t)$ holds true.

We point out that the set $\{t>0: \alpha(t)>0\}$ is open, by the continuity of $\alpha$; so that it is the union of at most countably many open intervals, say $I_{0} \bigcup\left(\bigcup_{i} I_{i}\right)$, where $I_{0}$ is of the form $(0, T)$ (obviously, it may occur that $I_{i}=\emptyset$ for all $i$ as well as $T=+\infty)$. Then it is straightforward to get

$$
\alpha^{\prime}(t)=\left(-\frac{N}{r}+g(t)\right) \chi_{\{\alpha(t)>0\}}(t)
$$


and so function $\alpha$ is absolutely continuous on each bounded interval. An important consequence of being $\alpha$ absolutely continuous is that equality $u^{\prime}(x, t)=$ $\alpha^{\prime}(t) \chi_{B_{r}(0)}(|x|)$ holds in the distributional sense and, arguing as in Remark 3.3, we obtain that $u^{\prime}$ is the time derivative of $u$ in the sense of Definition 2.7. Moreover, denoting by $T_{i}$ the lower extreme of interval $I_{i}$. it yields

$$
\begin{aligned}
\alpha(t)= & \left(\alpha_{0}-\frac{N}{r} t+\int_{0}^{t} g(\tau) d \tau\right) \chi_{I_{0}}(t) \\
& +\sum_{i}\left(-\frac{N}{r}\left(t-T_{i}\right)+\int_{T_{i}}^{t} g(\tau) d \tau\right) \chi_{I_{i}}(t)
\end{aligned}
$$

Therefore, the solution should be given by

$$
\begin{aligned}
u(x, t)= & \left(\alpha_{0}-\frac{N}{r} t+\int_{0}^{t} g(\tau) d \tau\right) \chi_{I_{0}}(t) \chi_{B_{r}(0)}(|x|) \\
& +\sum_{i}\left(-\frac{N}{r}\left(t-T_{i}\right)+\int_{T_{i}}^{t} g(\tau) d \tau\right) \chi_{I_{i}}(t) \chi_{B_{r}(0)}(|x|) .
\end{aligned}
$$

To go forward, we will consider the precise representative of $g: g(t)$ satisfies

$$
g(t)= \begin{cases}\lim _{\rho \rightarrow 0^{+}} \frac{1}{2 \rho} \int_{t-\rho}^{t+\rho} g(\tau) d \tau, & \text { if } t \in L ; \\ 0, & \text { if } t \notin L ;\end{cases}
$$

where $L$ denotes the set of Lebesgue points of $g$. Thus, $g(t)$ is well-defined for all $t>0$. Similarly, we may consider the precise representative of $\alpha^{\prime}$.

If $u$ is a solution, then there exists a radial vector field $\mathbf{z}$ satisfying

$$
u^{\prime}(t)=\operatorname{div} \mathbf{z}(t)+f(t)
$$

in $\mathcal{D}^{\prime}(\Omega)$ for almost all $t>0$. Thus, for almost all $t>0$, the divergence of $\mathbf{z}(t)$ is constant in $B_{r}(0)$ and vanishes in $B_{R}(0) \backslash B_{r}(0)$. A radial vector field $\mathbf{w}$ such that

$$
\operatorname{div} \mathbf{w}(x)= \begin{cases}-1, & \text { if }|x|<r \\ 0, & \text { if } r<|x|<R\end{cases}
$$

must be of the form $w(x)=\rho(|x|) x$, where $\rho$ satisfies

$$
|x| \rho^{\prime}(|x|)+N \rho(|x|)= \begin{cases}-1, & \text { if }|x|<r \\ 0, & \text { if } r<|x|<R .\end{cases}
$$

Solving this ODE, we deduce that

$$
\mathbf{w}(x)= \begin{cases}-\frac{x}{N}, & \text { if }|x|<r \\ -r^{N} \frac{x}{N|x|^{N}}, & \text { if } r<|x|<R .\end{cases}
$$

It is straightforward that $\|\mathbf{w}\|_{\infty} \leq \frac{r}{N}$ and its distributional divergence is really given by $\operatorname{div} \mathbf{w}(x, t)=-\chi_{B_{r}(0)}(|x|)$. Therefore, we look for a vector field satisfying

$$
\mathbf{z}(x, t)=\beta(t) \mathbf{w}(x)
$$


with $|\beta(t)| \leq \frac{N}{r}$ for almost all $t>0$. Let's determine the precise representative of $\beta$ : It follows from

$$
u^{\prime}(t)=\operatorname{div} \mathbf{z}(t)+f(t), \quad \text { in } \mathcal{D}^{\prime}(\Omega),
$$

that

$$
\alpha^{\prime}(t)=-\beta(t)+g(t) .
$$

Two cases must be considered. When $\alpha(t)>0$, we deduce from (34) and (36) that $\beta(t)=\frac{N}{r}$. On the other hand, if $\alpha(t)=\alpha^{\prime}(t)=0$ and $t$ is a Lebesgue point of $\alpha^{\prime}$, then (36) implies $g(t)=\beta(t) \leq \frac{N}{r}$. The conclusion is

$$
\alpha^{\prime}(t)=\left(-\frac{N}{r}+g(t)\right)^{+}
$$

for almost all $t>0$. It may happen that $t$ is not a Lebesgue point of $\alpha^{\prime}$, but still be an interesting case, namely: when $t=T_{i}$ for certain $i$. Then we have $\alpha_{+}^{\prime}\left(T_{i}\right)>0, \alpha\left(T_{i}\right)=0$ and $\alpha\left(T_{i}+\rho\right)>0$ for $\rho>0$ small enough; so that

$$
\alpha\left(T_{i}+\rho\right)=-\frac{N}{r} \rho+\int_{T_{i}}^{T_{i}+\rho} g(\tau) d \tau
$$

for $\rho>0$ small enough. Hence,

$$
\alpha_{+}^{\prime}\left(T_{i}\right)=\lim _{\rho \rightarrow 0^{+}} \frac{\alpha\left(T_{i}+\rho\right)}{\rho}=-\frac{N}{r}+g\left(T_{i}\right),
$$

and consequently $\beta\left(T_{i}\right)=-\frac{N}{r}$ and $g\left(T_{i}\right)>\frac{N}{r}$. By the way, this is the condition that must be satisfied by $g\left(T_{i}\right)$ for the elevation of $\alpha$.

In order to see that $u$, as defined in (35), is the actual solution to our problem, we have considered the vector field defined by

$$
\mathbf{z}(x, t)= \begin{cases}\frac{N}{r} \mathbf{w}(x), & \text { if } \alpha(t)>0 ; \\ g(t) \frac{N}{r} \mathbf{w}(x), & \text { if } \alpha(t)=0 .\end{cases}
$$

Since $\operatorname{div} \mathbf{z} \in L^{\infty}(\Omega \times(0,+\infty))$, it follows from [7] that $(\mathbf{z}(t), D v)$ is a Radon measure for almost all $t>0$ and all $v \in B V(\Omega)$. Furthermore, a Green's formula holds.

To see (8), we just have to verify that $u^{\prime}(t)=\operatorname{div} \mathbf{z}(t)+f(t)$ holds in the sense of distributions for almost all $t>0$. In fact, due to (37), if $\alpha^{\prime}(t)>0$, then $\alpha^{\prime}(t)=-\frac{N}{r}+g(t)$ holds, and if $\alpha^{\prime}(t)=0$, then $0=-g(t)+g(t)$ is obviously satisfied.

To check (9), fix $t>0$ such that $\alpha(t)>0$ and $\phi \in C_{0}^{\infty}(\Omega)$. Then

$$
\begin{aligned}
& \int_{\Omega} \phi(\mathbf{z}(t), D u(t))=-\int_{\Omega} u \mathbf{z} \cdot \nabla \phi-\int_{\Omega} u \phi \operatorname{div} \mathbf{z} \\
& \quad=\int_{\{|x| \leq r\}} \alpha(t) \frac{x}{r} \cdot \nabla \phi+\frac{N}{r} \int_{\{|x| \leq r\}} \alpha(t) \phi=\alpha(t) \int_{\{|x|=r\}} \phi=\int_{\Omega} \phi|D u(t)| .
\end{aligned}
$$

On the other hand, when $t>0$ satisfies $\alpha(t)=0$,

$$
(\mathbf{z}(t), D u(t))=0=\alpha(t) \chi_{B_{r}(0)}(|x|)=|D u(t)| .
$$

In any case, it yields $(\mathbf{z}(t), D u(t))=|D u(t)|$ as measures. 
Since $u(x)=0$ for $|x|=R$, condition (10) is straightforward. Therefore, $u$ is the solution to problem (1).

Several concluding remarks are in order:

Remark 7.1. By Theorem 6.1, we obtain some consequences on summability:

1. $g \in L^{1}(0,+\infty)$ implies $\alpha \in L^{1}(0,+\infty)$.

2. $g \in L^{\infty}(0,+\infty)$ implies $\alpha \in L^{\infty}(0,+\infty)$ and Lipschitz-continuous.

Furthermore, the proof of Theorem 4.1 implies that if $g \in L_{l o c}^{2}(0,+\infty)$, then $\alpha^{\prime} \in L^{2}(\delta, T)$ for all $0<\delta<T<\infty$.

Remark 7.2. $\quad$ 1. In the homogeneous problem, there exists a finite extinction time (see [3]). It would seem that $g>0$ prevents extinction in finite time, but this fact is not always true. For instance, if $g \in L^{\infty}(0,+\infty)$ and $\|g\|_{\infty}<\frac{N}{r}$, then choosing $T$ satisfying $\alpha_{0}+\left(\|g\|_{\infty}-\frac{N}{r}\right) T=0$ we obtain $\alpha(t)=0$ for all $t \geq T$.

2. By Theorem 6.2 , if $f \in L^{\infty}\left(0,+\infty ; W^{-1, \infty}(\Omega)\right)$ is such that its norm satisfies $\|f\|_{L^{\infty}\left(0,+\infty ; W^{-1, \infty}(\Omega)\right)} \leq 1$, then function $\alpha$ is nonincreasing, that is,

$$
\alpha^{\prime}(t)=-\frac{N}{r}+g(t) \leq 0, \quad t>0 .
$$

This implies $\|g\|_{\infty} \leq \frac{N}{r}$.

\section{Acknowledgments}

S. Segura de León has partially been supported by Ministerio de Economía y Competitividad (Spain) under project MTM2012-31103 and the second author was supported by Capes (Brazil) under project BEX 10053-12-3. They would like to thank J.M. Mazón for helping to make the realization of this project possible. Finally, they also want to thank the referee for her/his useful comments that have improved this paper.

\section{References}

[1] Ambrosio, L., Fusco, N., Pallara, D.: Functions of Bounded Variation and Free Discontinuity Problems, Oxford Mathematical Monographs (2000)

[2] Andreu, F., Ballester, C., Caselles, V., Mazón, J.M.: The Dirichlet problem for the total variational flow. J. Funct. Anal. 180, 347-403 (2001)

[3] Andreu, F., Caselles, V., Díaz, J.I., Mazón, J.M.: Some qualitative properties for the total variation flow. J. Funct. Anal. 188(2), 516-547 (2002)

[4] Andreu, F., Caselles, V., Mazón, J.M.: Existence and uniqueness of a solution for a parabolic quasilinear problem for linear growth functionals with $L^{1}$ data. Math. Ann. 322(1), 139-206 (2002) 
[5] Andreu, F., Caselles, V., Mazón, J.M.: Parabolic Quasilinear Equations Minimizing Linear Growth Functionals, Progress in Mathematics, vol. 223. Birkhauser, Basel (2004)

[6] Andreu, F., Caselles, V., Mazón, J.M., Moll, J.S.: The minimizing total variation flow with measure initial conditions. Commun. Contemp. Math. 6(3), 431$494(2004)$

[7] Anzellotti, G.: Pairings between measures and bounded functions and compensated compactness. Ann. di Matematica Pura Ed Appl. IV 135, 293-318 (1983)

[8] Ballester, C., Caselles, V., Verdera, J.: Disocclusion by joint interpolation of vector fields and gray levels. Multiscale Model. Simul. 2(1), 80-123 (2003)

[9] Bellettini, G., Caselles, V., Novaga, M.: The total variation flow in $R^{N}$. J. Differ. Equ. 184(2), 475-525 (2002)

[10] Chen, G.-Q., Frid, H.: Divergence-measure fields and hyperbolic conservation laws. Arch. Ration. Mech. Anal. 147, 89-118 (1999)

[11] Evans, L.C.: Partial Differential Equations, Graduate Studies in Mathematics, vol. 19. American Mathematical Society, Providence (1998)

[12] Evans, L.C., Gariepy, R.F.: Measure Theory and Fine Properties of Functions, Studies in Advanced Mathematics. CRC Press, Boca Raton (1992)

[13] Hardt, R., Zhou, X.: An evolution problem for linear growth functionals. Comm. Partial Differ. Equ. 19, 1879-1907 (1984)

[14] Landes, R.: On the existence of weak solutions for quasilinear parabolic boundary value problems. Proc. R. Soc. Edinb. Sect. A 89, 217-237 (1981)

[15] Landes, R., Mustonen, V.: On parabolic initial-boundary value problems with critical growth for the gradient. Ann. Inst. H. Poincaré Anal. Non Linéaire 11, 135-158 (1994)

[16] Lions, J.-L.: Quelques méthodes de résolution des problèmes aux limites non linéaires. Dunod; Gauthier-Villars, Paris (1969)

[17] Mercaldo, A., Segura de León, S., Trombetti, C.: On the behaviour of the solutions to $p$-Laplacian equations as $p$ goes to 1. Publ. Mat. 52(2), 377-411 (2008)

[18] Ou Yang, L.: The boundedness of solutions of linear differential equations $y^{\prime \prime}+$ $A(t) y=0$. Adv. Math. 3, 409-415 (1957)

[19] Schwartz, L.: Fonctions mesurables et ${ }^{*}$-scalairement mesurables, mesures banachiques majorées, martingales banachiques, et propriété de Radon-Nikodým, Séminaire d'analyse fonctionnelle (dit "Maurey-Schwartz") (E. Polytechnique) (1974-1975), exp. no. 4, pp. 1-17

[20] Simon, J.: Compact sets in the space $L^{p}(0, T ; B)$. Ann. Mat. Pura Appl. 146, 6596 (1987)

[21] Tölle, J.M.: Convergence of solutions to the $p$-Laplace evolution equation as $p$ goes to 1 (2011, preprint) 
[22] Webb, J.: An extension of Gronwall's inequality. Nonlinear Bound. Value Probl. 9, 196-204 (1999)

Sergio Segura de León

Departament d'Anàlisi Matemàtica

Universitat de València

C/Dr. Moliner 50

Burjassot 46100

Valencia

Spain

e-mail: sergio.segura@uv.es

Claudete M. Webler

Departamento de Matemática

Universidade Estadual de Maringá

Bloco F67, Avenida Colombo

5790 Campus Universitário

Maringá

Paraná 87020-900

Brazil

e-mail: cmwebler@uem.br

Received: 11 June 2014 .

Accepted: 9 March 2015. 